\title{
Genetic Based Discrete Particle Swarm Optimization for Elderly Day Care Center Timetabling
}

\author{
M.Y. LIN $^{1}$, K.S. CHIN ${ }^{2 *}$, and K.L. TSUI ${ }^{3}$, T.C. WONG ${ }^{4}$, \\ ${ }^{1}$ Department of Systems Engineering and Engineering Management, City University of Hong Kong, Kowloon Tong, \\ Kowloon, Hong Kong, meiyanlin2-c@my.cityu.edu.hk \\ ${ }^{2}$ Department of Systems Engineering and Engineering Management and Centre of Systems Informatics Engineering, \\ City University of Hong Kong, Kowloon, Hong Kong, mekschin@cityu.edu.hk \\ ${ }^{3}$ Department of Systems Engineering and Engineering Management and Centre of Systems Informatics Engineering, \\ City University of Hong Kong, Kowloon, Hong Kong, kltsui @ cityu.edu.hk \\ ${ }^{4}$ Department of Design, Manufacture, and Engineering Management, University of Strathclyde, Glasgow, Scotland, \\ United Kingdom, andywtc@graduate.hku.hk
}

\begin{abstract}
The timetabling problem of local Elderly Day Care Centers (EDCCs) is formulated into a weighted maximum constraint satisfaction problem (Max-CSP) in this study. The EDCC timetabling problem is a multi-dimensional assignment problem, where users (elderly) are required to perform activities that require different venues and timeslots, depending on operational constraints. These constraints are categorized into two: hard constraints, which must be fulfilled strictly, and soft constraints, which may be violated but with a penalty. Numerous methods have been successfully applied to the weighted Max-CSP; these methods include exact algorithms based on branch and bound techniques and approximation methods based on repair heuristics, such as the min-conflict heuristic. This study aims to explore the potential of evolutionary algorithms by proposing a genetic-based discrete particle swarm optimization (GDPSO) to solve the EDCC timetabling problem. The proposed method is compared with the min-conflict random-walk algorithm (MCRW), Tabu search (TS), standard particle swarm optimization (SPSO), and a guided genetic algorithm (GGA). Computational evidence shows that GDPSO significantly outperforms the other algorithms in terms of solution quality and efficiency.
\end{abstract}

\section{Keywords}

Timetabling problem, discrete particle swarm optimization, weighted Max-constraint satisfaction problem, Tabu search, genetic algorithm, min-conflict random walk 


\begin{tabular}{ll} 
Abbreviations & \\
ANOVA & Analysis Of Variance \\
BCO & Bee Colony Optimization \\
CSP & Constraint Satisfaction Problem \\
DPSO & Discrete Particle Swarm Optimization \\
EDCC & Elderly Day Care Center \\
FSP & Flow-Shop Scheduling Problem \\
GA & Genetic Algorithm \\
GDPSO & Genetic-based Discrete Particle Swarm Optimization \\
GGA & Guided Genetic Algorithm \\
JSP & Job Shop Scheduling Problem \\
Max-CSP & Maximum Constraint Satisfaction Problem \\
MCRW & Min-Conflict Random-Walk algorithm \\
NP-hard & non-deterministic polynomial hard \\
PSO & Particle Swarm Optimization \\
ROV & Ranked-Order Value \\
SA & Simulated Annealing \\
TS & Standard Particle Swarm Optimization \\
\hline
\end{tabular}




\section{Introduction}

Driven by fertility and mortality reduction, and medical and economic advancements, the rapid aging of the world population has been one of the major global demographic trends [1]. This trend has also increased the demand for age-friendly and affordable healthcare services, including the long-term care, curative care and preventive care. Thus, the quality of healthcare services provided by day-care centers, community care centers and nursing homes gains increasingly significant attention [2]. To ensure the quality of such services, centers should deliver more effective services. However, many care centers suffer from operational inefficiency. Driven by low resource utilization and long waiting lists from manual timetabling, the EDCCs in Hong Kong call for more studies to improve the quality of healthcare services.

The EDCC timetabling problem is the assignment of users (elderly) and the activities of these users to different venues and timeslots depending on the operational constraints of the day care center. Therefore, a feasible solution to this problem can be described by formulating a timetabling assignment which satisfies all hard constraints (constraints that should not be violated under any circumstance) and many soft constraints (constraints that may be violated but with a penalty). The infeasibility value of a solution is the sum of the number of hard constraint violations multiplied by a heavy penalty. Infeasibility value plus the sum of the number of soft constraints is the objective value. A solution is better than another solution if this solution has less objective value. The EDCC timetabling aims to find a feasible solution with smallest objective value and determine which soft constraints suffer the most violation. Timetabling problems are encountered in various situations, such as rostering duties of nurse in hospitals [3-7], scheduling transportation events [8], and constructing timetables for courses or examinations in the education industry [9-15]. The EDCC timetabling has some unique characteristics (e.g. first-come, first-served rule; different service center arrival patterns, and mixed event types in the same room) in contrast to other existing timetabling problems. The details of these differences are discussed in Section 2.1.

In addition to satisfying hard constraints, if the violations of soft constraints should be minimized, the EDCC timetabling can be defined as an optimization problem that seeks a solution that satisfies 
the maximum number of constraints and exposes the most violated constraints. Hence, it is formulated with respect to the Max-CSP framework [16]. A typical Max-CSP considers all constraints with a same weight, whereas it considers all soft constraints with a same weight but any hard constraint violation with a heavy penalty. The methods to Max-CSP include exact algorithms based on branch-and-bound techniques $[16,17]$ and approximation algorithms based on heuristics, such as the min-conflict [18] and TS [19]. Evolutionary algorithms, such as GA [20] and PSO [21] for solving Max-CSPs, have been examined because of their capacities to resolve successfully difficult problems in various domains.

This study presents a GDPSO to solve the EDCC timetabling problem under the Max-CSP framework. The PSO-based algorithm is proposed because of the following reasons.

- PSO-based algorithms are proven efficient and effective in solving many optimization problems, such as flow-shop scheduling (FSP) [22-26], timetabling [10, 13, 14], and vehicle routing [27, 28].

- PSO has several advantages, including a simple structure, flexibility (immediate accessibility for practical applications), easy implementation, rapid solution acquisition, and high robustness [24].

- An objective of the EDCC timetabling problem is to address the most violated constraints; PSO has been proven as promising in achieving it within a short time because PSO is a one-way information sharing mechanism, where only the local/global best particle provides the best information and all the particles tend to quickly converge into the best solution [29].

The proposed GDPSO is in the combination of min-conflict strategy, random walk, genetic operators and one-way information sharing mechanism from PSO. The min-conflict strategy gives greedy heuristic logic to search for better solutions in short time and random walk consists of a succession of random steps. Instead of using the standard update scheme of PSO, it applies the crossover and mutation operator cooperated with min-conflict strategy and random walk to update particles. Compared with swarm optimization algorithms, such as GGA [20] and SPSO [30], 
GDPSO has fewer parameters to be tuned and quick convergence. In contrast with heuristic methods, such as MCRW and TS [19], GDPSO has stable performance while MCRW may randomly work into a space that violate the hard constraints and provide an unfeasible solution. GDPSO also outperforms TS in terms of solution quality and convergence speed. The main contributions of this article are as follows:

- The presentation of a proposed GDPSO for the EDCC timetabling problem. The experimental results indicate that the proposed GDPSO is deemed superior over other benchmarking methods and also implies its potential in solving the Max-CSP.

- The description and implementation of a Max-CSP-based EDCC timetabling problem. It offers a clear knowledge about which constraint is the most violated one and give the HHC structure suggestions on improvement based on the solution details.

The remainder of this paper is organized as follows. Section 2 reviews the former studies on timetabling and PSO. Section 3 introduces the Max-CSP-based EDCC timetabling problem. Section 4 explains the rationale of the proposed GDPSO. Section 5 describes the experiment design. Finally, Section 6 concludes this study and recommends future research directions.

\section{Literature review}

\subsection{Timetabling problem}

Burke and Kingston [31] provided the following generic description of timetabling: "A timetabling problem is defined by four parameters: $\mathrm{T}$, a finite set of times; $\mathrm{R}$, a finite set of resources; $\mathrm{M}$, a finite set of meetings; and $\mathrm{C}$, a finite set of constraints. The problem is to assign times and resources to the meetings so as to satisfy the constraints as far as possible." Timetabling applications have been explored in various forms such as educational timetabling, nurse rostering, sports scheduling, and transportation timetabling.

Educational timetabling problems require the allocation of events to timetable periods while satisfying a set of hard constraints and minimizing a set of soft constraints [9-15]. Pillay [32] provided an overview of the research conducted in the school timetabling problem, which summarized its general definition and categorized constraints into seven groups (i.e. problem 
requirements, no clashes, resources utilization, workload, period distribution, preference and lesson constraints). University timetabling problem can be classified into course timetabling and examination timetabling, between which the substantial difference was summarized by MirHassani and Habibi [33]. For instance, a course has to be scheduled into exactly one room while several exams share a room or an exam split across several rooms. Critical discussions of the research on educational timetabling in last decades were presented in [32-34], which highlighted the new trends and key achievements.

Nurse rostering problems generate a schedule for each nurse, who has day off patterns, working shifts patterns and different work contracts, to fulfill the collective agreement requirements and hospital staffing demand coverage, while minimizing salary cost and maximizing nurse preferences and quality [3-7]. Burke and Curtois [6] developed a mathematical model for all the instances of nurse rostering problems by applying "regular expression" to incorporate their varying types of constraints (e.g., minimum/maximum consecutive work days, day on/off request, and shift on/off request). Solos et al. [3] proposed a more effective generic variable neighborhood search algorithm to solve seven different nurse rostering instances and summarize these varying types of constraints into two categories: hard constraints (e.g., all shift type demands must be met) and soft constraints (e.g., maximum number of hours worked), most of which were also modelled as an integer programming in [5] and included in [7] when presenting a mathematical formulation for all nurse rostering problem instances with 2 hard and 18 soft constraints in the First International Nurse Rostering Competition (INRC-2010). It is different from the educational timetabling problem mainly because of the demand coverage constraint, which specifies the number of nurses in each skill level [4], salary costs, nurse preferences, and degree of balance among nurses.

Furthermore, the main issue in sports scheduling is determining the date and venue for each tournament game. For example, a round robin tournament requires each team to play against all other teams in a fixed number of times. Moreover, breaks minimization, distance minimization, traveling tournament problem, and carry-over effects minimization can all be considered in sports scheduling, thus, this scheduling is different from the educational timetabling problem. Ribeiro [35] 
provided an introductory review of fundamental problems in sports scheduling and a survey of applications of optimization methods for solving them.

In terms of train timetabling problems, Cacchiani and Toth [8] presented an overview of the main works on train timetabling and distinguished it into the non-cyclic [36] and cyclic [37] version. Trains with cyclic timetables leave the stations at the beginning or at a specific interval every cycle. For example, if the cycle is one hour, trains leave the stations at the same minute every hour. For non-cyclic timetables, trains leave the stations depending on the latest traffic and passenger flows. Unlike cyclic timetables, non-cyclic timetable problems are more complex because the degree of freedom is higher when determining the train times. However, the train service can be more responsive simultaneously if the schedule is not fixed.

Given the distinct features of timetabling problems, different approaches have been proposed over the last few years. Mathematical approaches, such as integer programming, for formulating timetabling problems have been proposed by $[5,38]$. Timetabling have also employed heuristic methods, such as SA [12] and TS [9]; constraint-based methods [39] ; and population-based approaches, such as GA [11], BCO [15] and PSO [13, 14].

We introduce a different problem in this study, particularly, EDCC timetabling, which shares common characteristics with some of the exam timetabling problems, such as clashing, availability, and capacity constraints. The EDCC timetabling problem also has some unique characteristics that are quite different from the exam timetabling problem. Briefly, some of the unique features are as follows: the elderly reach a center through different means, which implies that the start time of users varies; the elderly who come first are served first; users cannot perform exercises/services in three consecutive timeslots (each timeslot is limited to 20 minutes) because of the users' health condition; and each elderly comes to the center with his/her own arrival pattern (e.g., Mon-Wed-Fri, Tue-Thu-Sat, or daily) each week. On one hand, the user satisfaction and resource utilization can be maximized by solving the EDCC problem. On the other hand, soft constraint violations help indicate the areas that need the most improvement. 


\subsection{Particle Swarm Optimization}

PSO is one of the latest evolutionary techniques to solve optimization problems. Based on the metaphor of social interaction and communication in a flock of birds or school of fishes, PSO was initially proposed by Eberhart and Kennedy [40] to optimize various continuous nonlinear functions. PSO has been efficiently applied to solve numerous combinatorial optimization problems, particularly scheduling and timetabling in actual instances, such as, FSP [22-26], home care worker visit scheduling [41], course timetabling in high schools or universities [10, 13, 14], and train service timetabling [29].

When PSO is used to solve scheduling problems, transformation methods, such as ROV [42], priority based [43], and heuristic assignment [41], have enabled "continuous" PSO to solve "discrete" combinatorial optimization problems. However, the transformation may require extra computational time in problem-solving. To avoid a tedious transformation, several researchers developed and applied different types of DPSO in scheduling problems. When designing DPSO for solving FSP, Ponnambalam et al. [24] presented particle by job permutation and velocity by being denoted as lists of moves to update particle; while Wang and Tang [25] also used the similar job permutation representation of particles. They defined three new operators (subtract, multiply, and add operators) to update the velocity and a self-adaptive perturbation strategy to update the particle position. The latter [25] turned out to outperform the former [24]. A hybrid algorithm combining DPSO simulated annealing was proposed by Shao et al. [26] to solve a multi-objective flexible JSP, and gave better results in terms of a number of Pareto solutions and computational time. They used operation permutation vector and machine allocation vector to present a solution and redefine the update schema of particles by applying a specific list of steps (e.g., calculating the similarity between current solution and personal best solution). For this promising potential of DPSO, Zhang et al. [23] developed an improved PSO algorithm with the particle as the permutations of all jobs and the velocity update model as a crossover operator cooperated by mutation operator, which obtained better performance and possesses better convergence property. Tseng and Liao [22] used several inheritance schemes motivated by GA, such as the one-point, two-point, and position-based inheritance for lot streaming FSP. They have reported that genetic operators based DPSO is very promising in solving scheduling problems. 
Therefore, we also propose a DPSO with genetic, mutation and crossover operators, whose details differ from those being applied in the literature because we have formulated the EDCC timetabling problem as a Max-CSP problem. More details are provided in the section 4.

PSOs are currently applied in solving the timetabling problems, by using some transformation methods, such as "round up or round off" and lists of moves (swap, insertion), to redefine update schema. Shiau [10] proposed a PSO incorporated with local search mechanism that explores a better solution improvement in university course timetabling problem. The study used the integer position values to round up these real values of particles to solve the conflict between the discrete solution space of the problem and a continuous space that particles search. The similar strategy (round off) was also used by Chen and Shih [14] when investigating PSO to solve the course timetabling problem. Similar to the representation of particles in a previous study [41], Tassopoulos and Beligiannis [13] presented particles in a two-dimensional array using rows and columns to represent class IDs and timeslots, respectively; By contrast of [41], they applied a "swap with probability" and "insertions of randomly selected timeslots" instead of velocity as applied in an SPSO. Given the absence of literature on DPSO in timetabling context, of which the update scheme is redefined by genetic operators, this study may provide useful insights into the potential application of the proposed GDPSO in solving timetabling problems.

The DPSO in literature with genetic operators has focused on identifying feasible solutions to specific scheduling/timetabling problem and does not monitor constraint violations when searching. In our Max-CSP-based timetabling problem, we aim to determine a feasible solution with the smallest objective value and address the most violated constraints. Therefore, a particle with fewer constraint violations in each of the particle dimensions should be selected to obtain particles with high constraints satisfaction at the end of the search process. Thus, an ancillary structure is proposed to store the cost of constraints violation for each GDPSO particle. The crossover and mutation operators developed by Bouamama et al. [20] are simplified and applied to our GDPSO and these operators are compared with other general crossover and mutation operators [22]. 


\section{EDCC timetabling problem}

\subsection{Problem description}

EDCCs usually provide different services, such as home healthcare, meal delivery, and day care services. Particularly, day care services include rehabilitation exercises (e.g. kicking sandbags and calculation training), social and recreational activities (e.g. group activities), and personal care (e.g. bathing). These services are provided in different rooms, and some of the activities require the help of assistants. Some key features of this EDCC are summarized as follows:

1. The elderly may reach and exit the center by shuttle buses or by themselves at certain periods. Hence, service must be provided to the elderly within the center, that is, to meet the service time windows. Given that the elderly have different arrival times, service is provided on a first-come-first-served basis. In addition to the arrival time, the elderly have different weekly arrival patterns corresponding to various clinical needs. For example, some may visit the center every day whereas some may only visit every Mon, Wed, and Fri or every Tue, Thu, and Sat. However, the elderly cannot do tasks in three consecutive timeslots (almost one hour) because of physical concerns.

2. The elderly must complete all assigned tasks at the center before leaving. These tasks include activities conducted in different available rooms, including bathing (personal care), group activity (social activity), and walking (recreational activity).

3. Each room has a maximum capacity, that is, the number of elderly in each room should not exceed the maximum capacity of the room during each timeslot.

4. Service is provided with specific equipment. The number of equipment for each exercise/service is fixed. Some services should be provided with the help of assistants. Different services can be provided in various rooms.

5. In each room, expect the bathroom, several types of services can be provided in the same timeslot. Assistants are present to provide help to the elderly. The number of assistants in each room is fixed, hence, the number of elderly who requires assistance in each room should not exceed the number of assistants in the room. 
6. Considering the health condition of the elderly, each timeslot is limited to 20 minutes, which is shorter than those in the educational timetabling problem. The number of timeslots in each working day can be calculated through the following equation: (end_time-start_time)/duration.

7. No restriction is imposed on the sequence of tasks for each elderly, that is, an activity can be performed as long as the elderly and associated resources are available in the same timeslot. The type and number of tasks for each elderly are designed based on his/her health condition upon admission, but these tasks are subject to change after future evaluation.

8. Some group activities need to be performed only at fixed timeslots on certain days because of some specific considerations, such as participant and room availabilities. An elderly who participates in a group activity cannot perform other tasks during the same timeslot.

The EDCC timetabling problem is coined based on the definition given in the study of [31]. Table 1 presents a list of constraints, including primary soft constraints and primary hard constraints. Table 2 presents a finite set of timeslots, resources and events. The problem is assigning timeslots and resources to events to satisfy constraints as much as possible. The weekly timetabling problem is divided into daily problems based on the arrival patterns of elderly.

Table 1. Primary constraints in EDCC Timetabling problem

\begin{tabular}{|l|l|}
\hline \multicolumn{2}{|c|}{ Primary hard constraints } \\
\hline 1 & Each elderly (user) cannot participate in more than one event simultaneously. \\
\hline 2 & Events/rooms compatibility. For instance, bathing is only done in the bath room; \\
\hline & \multicolumn{1}{|l|}{ Primary soft constraints } \\
\hline 2 & Similar services/exercises are required to be practiced in the same room. \\
\hline 3 & Some events share a room, but some specific events are performed in a specific room. \\
\hline 4 & The service for each elderly (users) should be provided within his/her time window. \\
\hline 5 & The elderly (users) should be scheduled considering the weekly arrival patterns. \\
\hline 6 & Group exercise/service should be performed at certain timeslots. \\
\hline 7 & Events should be scheduled as early as possible. \\
\hline 8 & Sufficient resources are assumed (room, equipments, assistant capacities). \\
\hline
\end{tabular}


Table 2. Notations

\begin{tabular}{|c|c|}
\hline M & Total number of activities (exercise and service) provided by the center \\
\hline $\mathrm{N}$ & Total number of the elderly (users) that comes to the center \\
\hline $\mathrm{K}$ & Total number of timeslots \\
\hline $\mathrm{n}$ & Total number of events \\
\hline Q & Total number of rooms \\
\hline $\mathrm{CA}_{\mathrm{a}}$ & Total number of equipment for a particular activity a \\
\hline $\mathrm{CR}_{\mathrm{j}}$ & Capacity of a room $\mathrm{j}$ \\
\hline CRA & Capacity of assistants \\
\hline $\mathrm{T}_{\mathrm{pb}}$ & First time slot that a user $\mathrm{p}$ can start doing an activity \\
\hline $\mathrm{T}_{\mathrm{pf}}$ & Last time slot that a user $\mathrm{p}$ can perform an activity \\
\hline $\mathrm{C}_{\mathrm{m}}$ & Constraint $\mathrm{m}$ \\
\hline $\mathrm{E}_{\mathrm{pa}}$ & Event describing that a user $\mathrm{p}$ has to perform an activity a \\
\hline S & Set of all activities provided by the center \\
\hline $\mathrm{P}$ & Set of users \\
\hline $\mathrm{T}$ & Set of daily time slots \\
\hline $\mathrm{R}$ & Set of rooms in the center \\
\hline $\mathrm{E}$ & Set of events that occur in a day \\
\hline $\mathrm{SA}_{\mathrm{a}}$ & Set of activities that require assistants \\
\hline$A_{j}$ & Set of activities that have to be performed in a room $j$ \\
\hline Gt & Set of time slots occupied by a group activity \\
\hline Pgroup & Set of elderly(users) who participate in a group activity \\
\hline$X$ & Set of variables in a constraint network \\
\hline $\mathrm{D}$ & Set of finite domains for the variables in a constraint network \\
\hline $\mathrm{C}$ & Set of constraints over the subset of variables in a constraint network \\
\hline
\end{tabular}

\subsection{Problem Formulation}

\subsubsection{Decision variables}

The key decision of our timetabling problem is to assign an event to a timeslot. Thus, the event-related decision variable is denoted as follows:

$$
x_{E_{p a} T_{t}}=\left\{\begin{array}{l}
1, \text { user } p \text { takes activity a }\left(E_{p a}\right) \text { at time } T_{t} ; \\
0, \text { otherwise. }
\end{array}\right.
$$




\subsubsection{Constraints}

Constraint (1) indicates that no user can perform more than one activity at a time.

$$
C_{1}: \quad \sum_{E_{p a} \in \mathrm{E}} x_{E_{p a} T_{t}}=1, \quad \forall \mathrm{p} \in \mathrm{P}, \forall \mathrm{T}_{\mathrm{t}} \in \mathrm{T}
$$

Constraint (2) presents that each event should be assigned to one timeslot.

$$
C_{2}: \quad \sum_{T_{t} \in T} x_{E_{p a} T_{t}}=1, \forall E_{p a} \in E
$$

Constraint (3) claims that users (who participate in the group activity) should not be assigned to timeslots occupied by a group activity.

$$
C_{3}: \quad \sum_{T_{t} \in G t} x_{E_{p a} T_{t}}=0, \forall p \in P_{\text {group }}, \forall E_{p a} \in E
$$

Constraint (4) makes sure that users should finish all of their pre-assigned activities during the time windows of users $\left[T_{p b}, T_{p f}\right]$.

$$
C_{4}: \quad \sum_{T_{t}=T_{p b}}^{T_{t}=T_{p f}} x_{E_{p a} T_{t}}=1, \quad \forall p \in P, \forall E_{p a} \in E
$$

Constraint (5) specifies that the number of users performing activities in the same timeslot cannot exceed the room capacity.

$$
C_{5}: \quad \sum_{a \in A_{j}} \sum_{E_{p a} \in E} x_{E_{p a} T_{t}} \leq C R_{j}, \forall T_{t} \in T, \forall j \in R
$$

Constraint (6) shows that the equipment of each activity is sufficient for the users at each timeslot.

$$
C_{6}: \quad \sum_{E_{p a} \in E} x_{E_{p a} T_{t}} \leq C A_{a}, \forall T_{t} \in T, \forall a \in S
$$

Constraint (7) points out that the number of assistants needed at the same timeslot should not exceed the capacity of that timeslot.

$$
C_{7}: \quad \sum_{a \in A_{s}, E_{p a} \in E} x_{E_{p a} T_{t}} \leq C R A, \forall T_{t} \in T
$$

Constraint (8) states that room $\mathrm{i}$ is closed in a period $\left[T_{s}, T_{e}\right]$ where $\mathrm{s}<\mathrm{e}$.

$$
C_{8}: \quad \sum_{T_{t}=T_{s}}^{T_{t}=T_{e}} x_{E_{p a} T_{t}}=0, \forall p \in P, \forall a \in A_{i}, \forall E_{p a} \in E, \forall i \in R
$$

Constraint (9) explains that the specific service $s_{j}$ for users should be assigned to timeslots that range from $\left[T_{a}, T_{b}\right]$.

$$
C_{9}: \quad \sum_{T_{t}=T_{a}}^{T_{t}=T_{b}} x_{E_{p s_{j}} T_{t}}=1, \forall E_{p s_{j}} \in E
$$

Constraints (1) to (3) are classified as hard constraints while the rest are soft constraints. Given that all hard constraints must be satisfied, we aim to identify the most violated soft constraints. 
These soft constraints may indicate the potential improvement for an EDCC if some of the constraints can be relaxed, for example, increasing the room capacity and the number of equipment, and extending the opening hours.

\subsubsection{Objective function}

The "daily" timetabling problem satisfies all hard constraints while minimizing the violations of soft constraints in each working day. Theoretically, the hard constraints can be violated but with a heavy penalty (W). Therefore, violation of hard constraints should be discouraged during the searching, and solutions with any hard constraint violation are regarded as infeasible. The objective function $f(s)$ for a timetable $s$ is the weighted sum of the number of violated hard constraints (the infeasibility value of timetable $s$ ) and violated soft constraints (the objective value of timetable $s$ ) [11], which is shown as follows:

$$
f(s)=\operatorname{hcv}(s) * W+\operatorname{scv}(s),
$$

where $h c v(s)$ is the number of hard constraint violations, $\operatorname{scv}(s)$ is the number of soft constraint violations in a timetable $s$, and $\boldsymbol{W}$ is a pre-determined large constant used to penalize (discourage) the violation of any hard constraints.

The essence of our timetabling problem is assigning events to timeslots, where events represent the users and their pre-assigned activities. The main idea of using "event" is that each user can only perform certain activities based on their health situation, rather than all activities that the center provides.

\subsection{Weighted Max-CSP-based EDCC Timetabling}

As an NP-hard problem, in general, weighted Max-CSP is significantly important in practice [19]. Given that all users have been assigned certain activities based on their health conditions when they are admitted, and certain activities are fixed in different rooms, the timetabling problem would be simplified as assigning the events to a certain timeslot subject to several constraints. Similar to CSP, the constraint network for the EDCC timetabling problem includes a finite set of events (a set $X$ of variables), a set of timeslots (a set $D$ of finite domains), and a set of constraints (a set $C$ of constraints over the subsets of $X$ ), and objective function described previously. 
The "daily" EDCC timetabling problem can be described under the framework of weighted Max-CSP, hence, the problem is also an NP-hard problem [44]. Weighted Max-CSP is generally defined as $(X, D, C)$, where $X=\left\{x_{1}, x_{2}, x_{3}, \ldots, x_{n}\right\}$ is the set of variables, $D=\left\{D_{1}, D_{2}, D_{3}, \ldots, D_{n}\right\}$ is the set of domains that contain the values a variable may be assigned to, and $C=$ $\left\{C_{1}, C_{2}, C_{3}, \ldots, C_{m}\right\}$ is the set of constraints over the subset of variables [16]. Max-CSP is an optimization problem of finding an assignment that satisfies as many constraints as possible. In many cases, weights are associated with constraints and the goal is to maximize the weighted sum of all satisfied constraints. Based on the Max-CSP framework, the "daily" EDCC timetabling problem is described as follows:

1. $X=\left\{x_{1}, x_{2}, x_{3}, \ldots, x_{n}\right\}$, where $n$ is the total number of events. Each variable represents one event. We denote the events with index by increasing order of users and activities, for example, two users $\left(p_{i}, p_{j}\right)$ are present; one has three tasks $\left(s_{o}, s_{p}, s_{q}\right)$ and the other also has three tasks $\left(s_{p}, s_{q}, s_{t}\right)$. Then, six events would be denoted as $x_{a}, x_{b}, x_{c}, x_{d}, x_{e}, x_{f}$, where $x_{a}$ represents the pair $\left\langle p_{i}, s_{o}\right\rangle, x_{b}$ represents the pair $\left\langle x_{i}, s_{p}\right\rangle$, and $x_{c}$ represents the pair $\left\langle p_{i}, s_{q}>\right.$, and so on.

2. $D=\left\{\mathrm{D}_{1}, \mathrm{D}_{2}, \mathrm{D}_{3}, \ldots, \mathrm{D}_{\mathrm{n}}\right\}$, where $\mathrm{D}_{1}=\mathrm{D}_{2}=\mathrm{D}_{3}, \ldots,=\mathrm{D}_{\mathrm{n}}=\{1,2,3, \ldots, K\}$, the available timeslots for each event in a single working day.

3. $C=\left\{\mathrm{C}_{1}, \mathrm{C}_{2}, \mathrm{C}_{3}, \ldots, \mathrm{C}_{\mathrm{m}}\right\}$, where $\mathrm{C}_{1}$ represents constraint (1), $\mathrm{C}_{2}$ represents constraint (2), and so on. For ease of presentation, all constraints will be presented in sets. For example, a hard constraint that describes no user can do more than one activity at the same time would be presented by $\mathrm{C}_{1}=\left\{\mathrm{C}_{11}, \mathrm{C}_{12}, \ldots, \mathrm{C}_{1 \mathrm{~N}}\right\}$, where $N$ is the total number of users. In other words, $\mathrm{C}_{1 \mathrm{i}}$ denotes the effect of constraint (1) on all events that involve user $\mathrm{i}$.

\section{The proposed GDPSO}

The proposed GDPSO uses an event-based representation of particles and genetic operator based update scheme with an ancillary structure (called "template" in this algorithm) to store the violation cost of constraints for each particle. New crossover and mutation operators that differ 
from that of conventional GAs are applied. The position of the $\boldsymbol{i}$-th particle is updated using Equation (16) at each iteration step, where NP is the size of the entire swarm.

$$
X_{i}^{t+1}=w \oplus F_{2}\left(F_{1}\left(F_{1}\left(X_{i}^{t}, P_{i}^{t}\right), G^{t}\right)\right), \forall i=1,2, \ldots, N P
$$

The update equation consists of three components. The first component denoted as $\lambda_{i}^{t}$ is $\mathrm{F}_{1}\left(\mathrm{X}_{\mathrm{i}}^{\mathrm{t}}, \mathrm{P}_{\mathrm{i}}^{\mathrm{t}}\right)$, where $\mathrm{P}_{\mathrm{i}}^{\mathrm{t}}$ is the personal best particle of particle $\boldsymbol{i}$. It is the cognition part of the particle to represent the private thinking of the particle itself, and $F_{1}$ represents the crossover operator presented by Bouamama et al. [20]. The second component is denoted as $\delta_{\mathrm{i}}^{\mathrm{t}}$ is $F_{1}\left(\lambda_{i}^{t}, G^{t}\right)$, where $G^{t}$ is the global best particle in generation $t$. It is the social part of the particle to represent the collaboration among particles. The third component is $\mathrm{X}_{\mathrm{i}}^{\mathrm{t}+1}=w \oplus \mathrm{F}_{2}\left(\delta_{\mathrm{i}}^{\mathrm{t}}\right)$, which presents the velocity of the particle, and $F_{2}$ represents the two different mutation operators with a probability of $w . \delta_{\mathrm{i}}^{\mathrm{t}}$ is either updated by guided mutation operator via the $\boldsymbol{i}$-th template or by random mutation operator depending on the choice of a uniform random number $w$ ranging [0, 1]. Figure 1 presents pseudo codes of $F_{1}, F_{2}$ and update functions. Algorithms 1 and 2 describe crossover and mutation operators of the update scheme, whereas the pseudo codes of the update scheme for particles is presented in Algorithms 3.

The similarities between SPSO and GPSO are the usage of update scheme that both use the information of global best particle of swarm and personal best particle of each particle, and the update scheme of the best position of each particle and global best position of swarm. The difference between SPSO and GPSO is the update scheme of the position of each particle and the velocity of each particle. In SPSO, it uses two equations to update the velocity and position accordingly, while the proposed GDPSO only use Equation (16), which conducts crossover and mutation operation with considering the problem characteristics and using min-conflict heuristic and random walk, while SPSO ignores the discrete characteristic of problem and considers it as a continuous one with the update scheme proposed in [30, 40]. Both GDPSO and GGA apply the crossover, mutation operator in algorithms, however, the difference is that GDPSO crossover each particle with global and personal best particles whereas GGA crossover two chromosomes selected by mating pools. The difference among GDPSO, TS and MCRW is that GDPSO store global and personal best information and share their information with each particle during each 
generation, MCRW only use the min-conflict and random walk to explore the space, while TS use neighborhood to store the information of potential space for exploration in future.

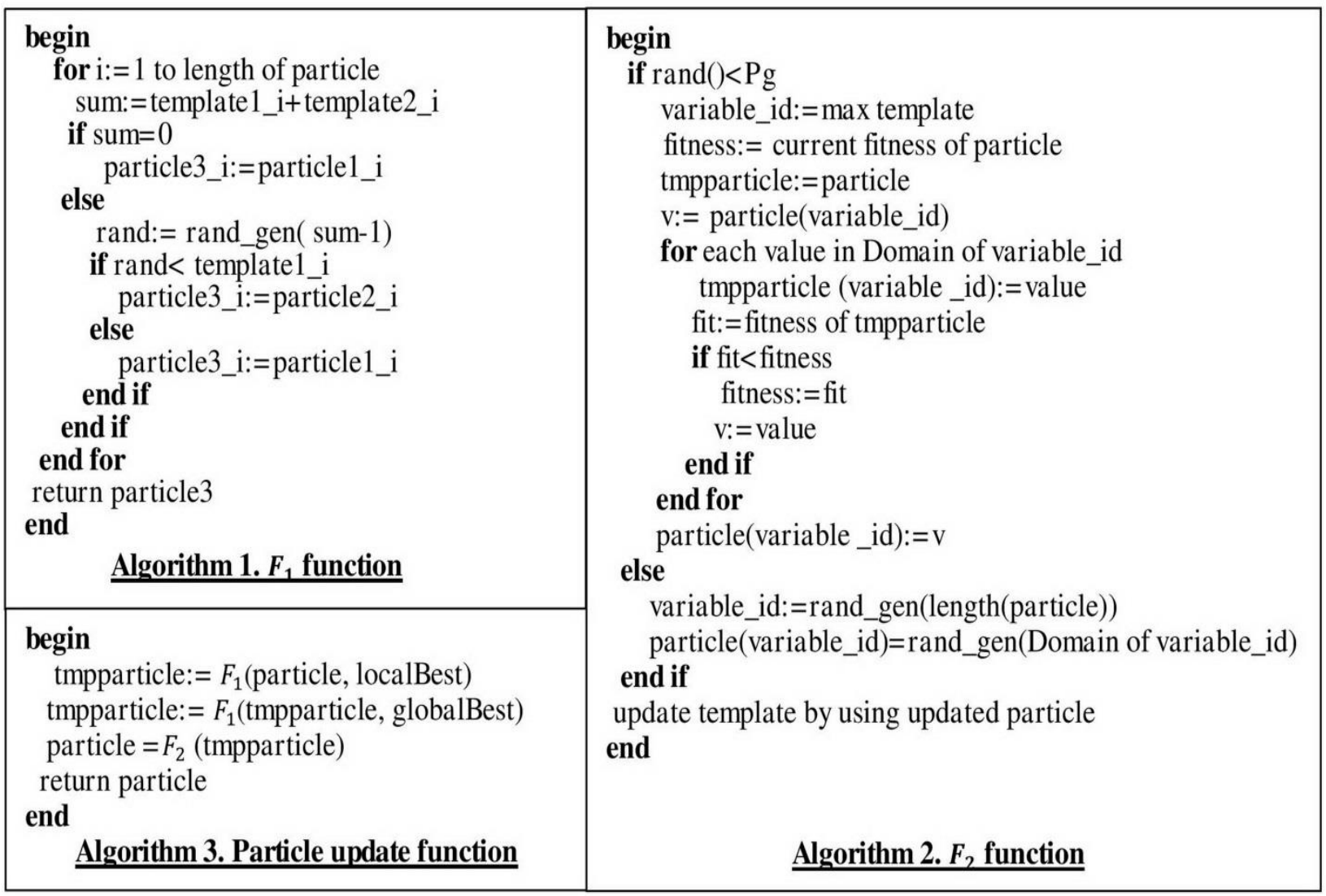

Figure 1. Pseudo codes of $F_{1}, F_{2}$ and update functions

\subsection{Particle representation}

The solution to the EDCC timetabling problem can be represented in the form of an order list of $t_{i}(\mathrm{i}$ $=1,2, \ldots, 19)$, in which the index of each $t_{i}$ is the identification number of an event $e_{i} \in \mathrm{E}(\mathrm{i}=1,2, \ldots$, $n$ ), where $n$ is the total number of events in a certain working day. The solution is as follows: the particle (vector) is composed of the daily assignment of all events to certain timeslots, which implies that the size of the particle is equal to the total number of events (Figure 2). We suppose that the $i$-th particle $X_{i}^{t}$ is presented in Figure 2 and the timeslots are allocated to $x_{i, j}^{t}$ (events, $j=1,2, \ldots, n)$ in an ordered list, such as $7,6,1, \ldots, 9,15,4$, in which timeslot 7 is allocated to $x_{i, 1}^{t}$ (i.e., event $\left.1<p_{3}, s_{12}\right\rangle$ of user id 3), given that user ids 1 and 2 are not scheduled on this working day, and timeslot 6 is allocated to $x_{i, 2}^{t}$ (event $2\left\langle p_{3,}, s_{25}>\right.$ of user id 3), and so on. 


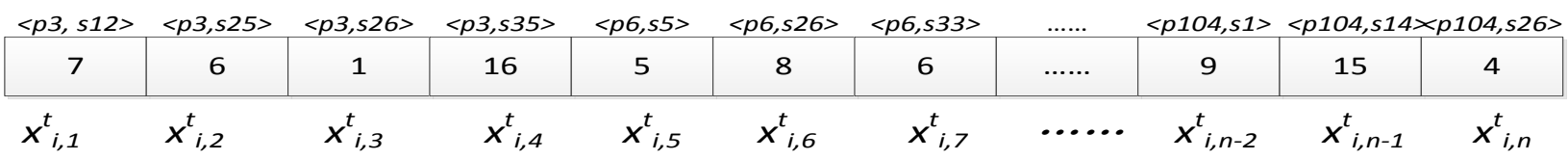

Figure 2. Representation of particle

Next, we have to check the violation of constraints associated with each of the particles. Since each cell of the particle denotes an assignment of one event to one timeslot, such assignment may or may not violate any constraints. If the highest cost of constraint violation is found for such assignment, the objective value of the particle may be significantly minimized by adjusting this particular assignment (i.e., allocate that event to another timeslot). Thus, we have to capture the cost of violation of constraints for all cells of a particle during the search process. This task involves attaching a new data structure called "template" to each particle (Figure 3). Suppose $\boldsymbol{i}$-th template for $\boldsymbol{i}$-th particle is denoted by $\operatorname{Temp}_{i}^{t}$, then $\operatorname{Temp}_{i, j}^{t}$ stores the total cost of constraints violated by $x_{i, j}^{t}$. This cost has two components, such as the number of hard constraint violations multiplied by a large constant (denoted by $\boldsymbol{W}$, say $10^{6}$ ) and the number of soft constraint violations multiplied by 1 . For ease of representation, the events are omitted from particle representation in the following parts. Only their notation $x_{i, j}^{t}$ is shown.

\begin{tabular}{|c|c|c|c|c|c|c|c|c|c|c|}
\hline$<p 3, s 12>$ & $<p 3, s 25\rangle$ & $<p 3, s 26>$ & $<p 3, s 35>$ & $\langle p 6, s 5\rangle$ & $<p 6, s 26>$ & $<p 6, s 33>$ & $\ldots \ldots$ & $\langle p 104, s 1\rangle$ & $<p 104, s$ & $104, s 26>$ \\
\hline 2 & 1 & 1 & 4 & 3 & 1 & 2 & $\ldots \ldots$ & 1 & 10 & 7 \\
\hline
\end{tabular}

Figure 3. Representation of template attached to each particle

GDPSO randomly initializes $N P$ particles to generate an initial swarm in which $N P$ is the swarm size. Before commencing the optimization process, GDPSO initializes all "templates" that correspond to its particles. These particles are modified iteratively based on collective experiences to improve their solution quality, and then their corresponding "templates" are updated.

\subsection{Novel crossover operator $F_{1}$}

The crossover operator of our GDPSO is illustrated in Figure 4. Two particles, $\lambda_{i}^{t}$ and $P_{i}^{t}(i$-th particle and its previous best local position, $\boldsymbol{i}=1, \ldots, N P)$, are crossed in each iteration. The "templates" for $\lambda_{i}^{t}$ and $\mathrm{P}_{\mathrm{i}}^{\mathrm{t}}$ are denoted by $\mathrm{T} \lambda_{\mathrm{i}}^{\mathrm{t}}$ and $\mathrm{TP}_{\mathrm{i}}^{\mathrm{t}}$ respectively and are used to generate the intermediate particle $\delta_{i}^{t}$. To generate $\delta_{i, j}^{t}$ for $\delta_{i}^{t}$, the sum of "template" $T \lambda_{i, j}^{t}$ and $\mathrm{TP}_{\mathrm{i}, \mathrm{j}}^{\mathrm{t}}$ is 
calculated and denoted by Sum. If Sum is equal to 0 (both $T \lambda_{i, j}^{t}$ and $\mathrm{TP}_{\mathrm{i}, \mathrm{j}}^{\mathrm{t}}$ are 0 ), then $\lambda_{\mathrm{i}, \mathrm{j}}^{\mathrm{t}}$ is assigned to $\delta_{\mathrm{i}, \mathrm{j}}^{\mathrm{t}}$; otherwise, we generate a random integer number (denoted by $\boldsymbol{R a n I}$ ) between 0 and $\boldsymbol{S u m}$-1. If $\operatorname{RanI}$ is less than $T \lambda_{\mathrm{i}, \mathrm{j}}^{\mathrm{t}}$, then the value of $\mathrm{P}_{\mathrm{i}, \mathrm{j}}^{\mathrm{t}}$ is assigned to $\delta_{\mathrm{i}, \mathrm{j}}^{\mathrm{t}}$; otherwise, we assign $\lambda_{i, j}^{t}$ to $\delta_{i, j}^{t}$, as indicated in Figure 4. This process continues until $\delta_{i, n}^{t}$ has been assigned. When processing is completed, the intermediate particle $\delta_{i}^{t}$ would be crossed with $G^{t}$ (the global best position in the current generation). The cross procedure is similar to that of $\lambda_{i}^{t}$ and $\mathrm{P}_{\mathrm{i}}^{\mathrm{t}}$.

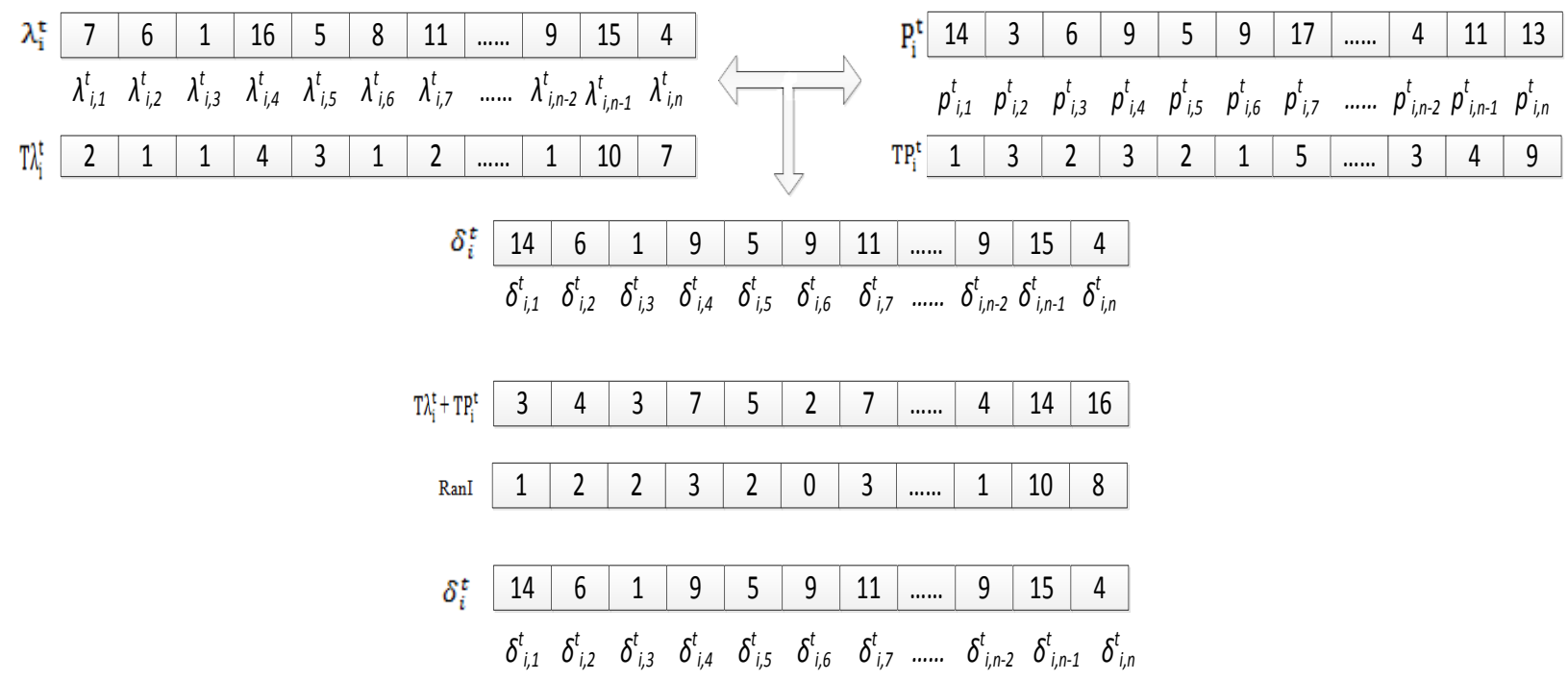

Figure 4. Crossover operator applied to $\lambda_{i}^{t}$ and $P_{i}^{t}$

\subsection{Mutation operator $F_{2}$}

GDPSO adopts two different mutation operators. The first one is a randomly selected single cell (i.e., the mutation point). The value of that cell is randomly assigned a timeslot between 1 and 19 (Figure 5). The cell explores new spaces, thereby resulting in a significant degree of genetic variation [45]. However, violation of constraints would possibly increase for the mutated particle because of the randomness. To remedy this drawback, another mutation operator is considered to supplement the random mutation. The cell (mutation point) of the particle is now selected according to its corresponding "template." For example, we suppose that the number of constraints violated by $\mathrm{x}_{i, n-1}^{\mathrm{t}}$ in Figure 6 is the largest in the $\boldsymbol{i}$-th template, then we select $\mathrm{x}_{i, n-1}^{\mathrm{t}}$ in the $\boldsymbol{i}$-th particle and update its value using the min-conflict heuristic method. This heuristic method tests all possible values that $\mathrm{x}_{i, n-1}^{\mathrm{t}}$ can be assigned to, and selects the best value for $\mathrm{x}_{i, n-1}^{\mathrm{t}}$ by comparing the objective value among all of these possible assignments. 


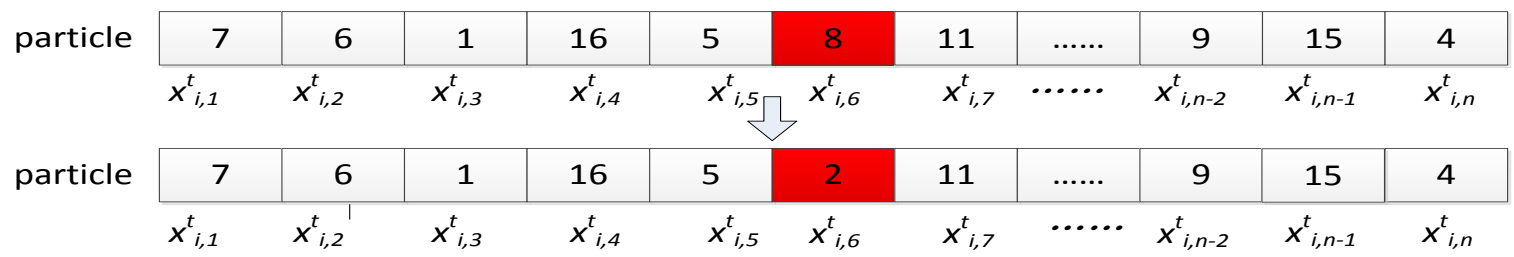

Figure 5.Mutation operator with random selected $\mathbf{j}$ for $X_{i}^{t}$

\begin{tabular}{|c|c|c|c|c|c|c|c|c|c|c|c|}
\hline \multirow[t]{2}{*}{ particle } & 7 & 6 & 1 & 16 & 5 & 8 & 11 & $\ldots \ldots$ & 9 & 15 & 4 \\
\hline & $x_{i, 1}^{t}$ & $x_{i, 2}^{t}$ & $x_{i, 3}^{t}$ & $x_{i, 4}^{t}$ & $x_{i, 5}^{t}$ & $x_{i, 6}^{t}$ & $x_{i, 7}^{t}$ & $\cdots \cdots$ & $x_{i, n-2}^{t}$ & $x_{i, n-1}^{t}$ & $x_{i, n}^{t}$ \\
\hline template & 2 & 1 & 1 & 4 & 3 & 1 & 2 & $\ldots \ldots$ & 1 & 10 & 7 \\
\hline \multirow{3}{*}{ particle } & \multicolumn{11}{|c|}{ 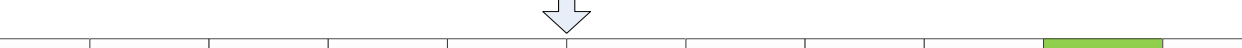 } \\
\hline & $\gamma$ & 0 & 1 & 10 & 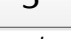 & 0 & $\perp \perp$ & $\ldots \ldots$ & 9 & 0 & 4 \\
\hline & $x_{i, 1}^{t}$ & $x_{i, 2}^{t}$ & $x_{i, 3}^{t}$ & $x_{i, 4}^{t}$ & $x_{i, 5}^{t}$ & $x_{i, 6}^{t}$ & $x_{i, 7}^{t}$ & \multicolumn{2}{|c|}{$\cdots \cdots x_{i, n-2}^{t}$} & $x_{i, n-1}^{t}$ & $x_{i, n}^{t}$ \\
\hline template & 1 & 2 & 1 & 2 & 5 & 2 & 7 & $\ldots \ldots$ & 2 & 1 & 2 \\
\hline
\end{tabular}

Figure 6.Mutation operator with min-conflict heuristic

\subsection{Fitness evaluation}

As defined by Equation (10), the objective is to minimize the violation of hard and as many soft constraints as possible. The fitness evaluation uses the objective function to calculate the fitness of each particle, which implies that smaller fitness (smaller constraint violation cost) of a particle results in a better solution.

\subsection{DPSO with other genetic operators}

To justify our proposed GDPSO, we examine three DPSO methods with other genetic operators. We denote DPSO1 as the one-point crossover operator, DPSO2 as two-point crossover operator I, and DPSO3 as two-point crossover operator II [22]. The main difference among the proposed GDPSO, DPSO1, DPSO2, and DPSO3 is the crossover operator (Figures 7 to 9). DPSO 1 to 3 randomly select the crossover point and do not adopt the ancillary structure used in our proposed GDPSO. These DPSO-based methods are compared to evaluate the performance of our GDPSO.

\begin{tabular}{|c|c|c|c|c|c|c|c|c|c|c|c|}
\hline \multirow[b]{2}{*}{$x_{i}$} & \multicolumn{11}{|c|}{ crossover point } \\
\hline & 7 & 6 & 1 & 16 & 5 & 8 & 11 & ....... & 9 & 15 & 4 \\
\hline & $\lambda_{\mathrm{i}, 1}^{\mathrm{t}}$ & $\lambda^{t}{ }_{i, 2}$ & $\lambda^{t}{ }_{i, 3}$ & $\lambda^{t}{ }_{i, 4}$ & $\lambda^{t}{ }_{i, 5}$ & $\lambda^{t}{ }_{i, 6}$ & $\lambda^{t}{ }_{i, 7}$ & ...... & $\lambda_{i, n-2}^{t}$ & $\lambda_{i, n-1}^{t}$ & $\lambda^{t}{ }_{i, n}$ \\
\hline \multirow[t]{2}{*}{$\mathbf{P}_{i}^{t}$} & 14 & 3 & 6 & 9 & 5 & 9 & 17 & $\ldots \ldots$ & 4 & 11 & 13 \\
\hline & $p^{t}{ }_{i, 1}$ & $p^{t}{ }_{i, 2}$ & $p^{t}{ }_{i, 3}$ & $p^{t}{ }_{i, 4}$ & $p^{t}{ }_{i, 5}$ & $p^{t}{ }_{i, 6}$ & $p^{t}{ }_{i, 7}$ & $\ldots .$. & $p^{t}{ }_{i, n-2}$ & $p_{i, n-1}^{t}$ & $p^{t}{ }_{i, n}$ \\
\hline \multirow[t]{2}{*}{$b^{2}$} & 7 & 6 & 1 & 16 & 5 & 8 & 17 & ....... & 4 & 11 & 13 \\
\hline & $\delta^{t}{ }_{i, 1}$ & $\delta^{t}{ }_{i, 2}$ & $\delta^{t}{ }_{i, 3}$ & $\delta_{i, 4}^{t}$ & $\delta^{t}{ }_{i, 5}$ & $\delta^{t}{ }_{i, \sigma}$ & $\delta_{i, \tau}^{t}$ & …... & $\delta_{i, n-2}^{t}$ & $\delta_{i, n-1}^{t}$ & $\delta_{i, n}^{t}$ \\
\hline
\end{tabular}

Figure 7. One-point crossover operator applied to $\lambda_{i}^{t}$ and $P_{i}^{t}$ 
First crossover point Second crossover point

\begin{tabular}{|c|c|c|c|c|c|c|c|c|c|c|c|}
\hline \multirow[t]{2}{*}{$\lambda_{i}^{\pi}$} & 7 & 6 & 1 & 16 & 5 & 8 & 11 & $\ldots . .$. & 9 & 15 & 4 \\
\hline & $\lambda_{i, 1}^{t}$ & $\lambda_{i, 2}^{t}$ & $\lambda_{i, 3}^{t}$ & $\lambda_{i, 4}^{t}$ & $\lambda_{i, 5}^{t}$ & $\lambda_{i, 6}^{t}$ & $\lambda_{i, 7}^{t}$ & ...... & $\lambda_{i, n-2}^{t}$ & $\lambda_{i, n-1}^{t}$ & $\lambda_{i, n}^{t}$ \\
\hline \multirow[t]{2}{*}{$P_{i}^{t}$} & 14 & 3 & 6 & 9 & 5 & 9 & 17 & $\ldots \ldots$ & 4 & 11 & 13 \\
\hline & $p_{i, 1}^{t}$ & $p_{i, 2}^{t}$ & $p_{i, 3}^{t}$ & $\mathrm{p}_{\mathrm{i}, 4}^{\mathrm{t}}$ & $p_{i, 5}^{t}$ & $p_{i, 6}^{t}$ & $p_{i, 7}^{t}$ & ...... & $p_{i, n-2}^{t}$ & $p_{i, n-1}^{t}$ & $p^{t}{ }_{i, n}$ \\
\hline$\delta_{i}^{t}$ & 7 & 6 & 1 & 16 & 5 & 8 & 17 & $\cdots \cdots$ & 9 & 15 & 4 \\
\hline
\end{tabular}

Figure 8. Two-point crossover operator $I$ applied to $\lambda_{i}^{t}$ and $P_{i}^{t}$

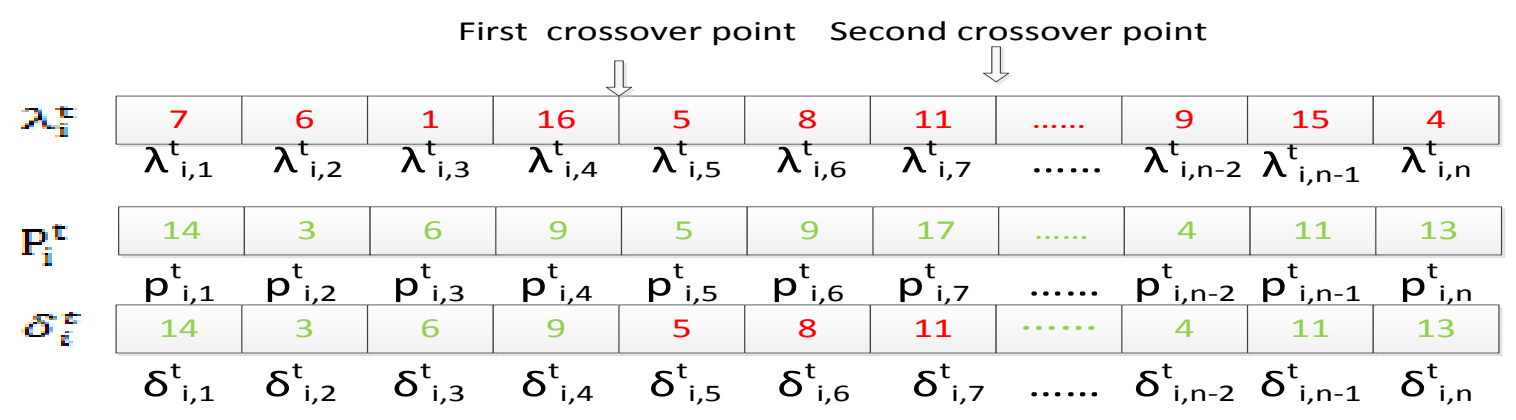

Figure 9. Two-point crossover operator II applied to $\lambda_{i}^{t}$ and $P_{i}^{t}$

\section{Experimentation}

In addition to DPSO-based methods, two common methods to solve Max-CSP are examined, namely, TS and MCRW [19]. Since the timetabling problem in this study is based on the framework of Max-CSP, TS and MCRW are compared with our GDPSO. Moreover, the GGA modified from the distributed guide genetic algorithm [20] is compared with our GDPSO because both algorithms adopt the similar scheme of crossover and mutation. Our GDPSO is also benchmarked with the SPSO without any transformation method.

Our proposed GDPSO, SPSO [30], DPSO 1 to 3 [22], TS [19], MCRW [19], and GGA [20] are implemented in Matlab R2011b and tested on a personal computer with Intel $3.30 \mathrm{GHz}, 3.2 \mathrm{~GB}$ usable memory, and Windows 7 operating system.

\subsection{Data sets}

Table 3 shows all of the constraints of EDCC for a week after raw data processing. In this table, $C_{i}$ represents constraint (i) described in Section 3.2.2, except constraint (2), which is always satisfied by allocating events to any timeslot. Column " $\mathrm{C}_{8 \sim 9}$ " denote the sum of constraints in relation to $C_{8}$ and $C_{9}$. Columns "Hard" and "Soft" show the total number of hard constraints (i.e., 
$C_{1}$ and $C_{3}$ ) and that of soft constraints (i.e., $C_{4} \sim C_{9}$ ) for each working day. Column "Total" indicates the sum of hard and soft constraints for each working day.

Table 3. Number of constraints of EDCC timetabling (one week)

\begin{tabular}{|c|c|c|c|c|c|c|c|c|c|c|}
\hline Case & $\mathbf{C}_{\mathbf{1}}$ & $\mathbf{C}_{\mathbf{3}}$ & $\mathbf{C}_{\mathbf{4}}$ & $\mathbf{C}_{\mathbf{5}}$ & $\mathbf{C}_{\mathbf{6}}$ & $\mathbf{C}_{\mathbf{7}}$ & $\mathbf{C}_{\mathbf{8 - 9}}$ & Hard & Soft & Total \\
\hline Mon & 67 & 6 & 218 & 2 & 25 & 1 & 215 & 73 & 461 & 534 \\
\hline Tue & 65 & 16 & 206 & 2 & 28 & 1 & 202 & 81 & 439 & 520 \\
\hline Wed & 69 & 6 & 227 & 2 & 26 & 1 & 224 & 75 & 480 & 555 \\
\hline Thu & 63 & 8 & 198 & 2 & 27 & 1 & 195 & 71 & 423 & 494 \\
\hline Fri & 69 & 14 & 227 & 2 & 25 & 1 & 224 & 83 & 479 & 562 \\
\hline Sat & 59 & 7 & 184 & 2 & 26 & 1 & 181 & 66 & 394 & 460 \\
\hline
\end{tabular}

\subsection{Evaluation of GDPSO}

Three experiments were conducted to evaluate the performance of our proposed GDPSO. The first experiment analyzed the sensitivity of the GDPSO to its key parameters (i.e., $N P, N G$, and $p_{g}$ ) for our EDCC timetabling problem. Similar experiments were also conducted to tune the parameters of GGA, SPSO, MCRW, TS, and DPSO1 to 3 to ensure that the benchmarking was equitable. The second experiment compared the performance of different genetic operators (including our novel genetic operators) that can be used in DPSO. The third experiment compared the performance of our GDPSO, GGA, SPSO, MCRW, and TS. Similar raw data and objective function were used to evaluate all methods. In order to obtain the mean fitness and observe the stability of each algorithm, each algorithm ran 20 times under same parameters setting for each working day in all three experiments.

\subsubsection{First Experiment}

\subsubsection{Sensitivity analysis of GDPSO}

To improve our GDPSO design, preliminary tests were conducted on the data sets. Our GDPSO has three key parameters, namely, population size $(N P)$, generation number $(N G)$, and guide probability $\left(P_{g}\right)$. Since each parameter has three levels, a $3^{3}$ full factorial design described by Montgomery [46] can be used to analyze the sensitivity of GDPSO to its parameters. As suggested by [13], the $N P$ can be typically set from 20 to 40 . The larger the $N G$, the longer the computational time would be. Thus, the range of $N G$ is deliberately set to 20 to 80 . After several pre-tests, no significant difference is observed in performance when setting $N G=80$ against $N G>$ 
80. The range of $P_{g}$ is set to 0.3 to 0.8 because this parameter requires longer computing time when $P_{g}=1$. The performance $\left(P_{g}=1\right)$ is insignificantly different from that of $P_{g}=0.8$, but the performance $\left(P_{g}=0.3\right)$ is significantly different from that of $P_{g}=0$. Experimentally, three levels of each parameter $\{$ low, medium, high $\}$ are set as: $N P\{20,30,40\}, N G\{20,40,80\}$, and $P_{g}\{0.3$, $0.5,0.8\}$.

The ANOVA results are presented in Table 4. The table shows that NP, NG, $p_{g}$, and their interactions $N G^{*} P_{g}$ have a significant effect $(p=0)$ on GDPSO performance. To determine the appropriate values for each parameter, the main parameters and their interactions with significant effects are presented in Figure 10 in which the low level is denoted as 0, medium level as 1, and high level as 2. Figure 10 also contains the mean reference line of y-coordinate, which indicates the baseline of fitness selection so we only consider the parameters setting that makes fitness less than the mean value. Figure 10 indicates that the medium and high level of NG outperform low level of NG, medium level of NP performs better than low level of NP but performs a little bit worse than high level of NP, whereas high level of $P_{g}$ outperforms medium and low levels of $P_{g}$. When $N G$ is set at high level, the $P_{g}$ can be set at any level since performance is not so much different (the difference among them is less than 1), as indicated in Fig.10 (d). Because the larger $N G$ is, the longer computational time it will be, $N G$ is set to 40 . Although $N P$ has significant effects, for the sake of computational time, the experiment with medium $N P$ can help obtain slightly better results (making sure that the fitness is less than the mean value). Therefore, for our EDCC problem, the parameters are set to $N P=30, N G=40$, and $P_{g}=0.8$.

Table 4. ANOVA for parameters

\begin{tabular}{|c|c|c|c|c|c|}
\hline Source & $\begin{array}{c}\text { Sum of } \\
\text { Squares }\end{array}$ & df & $\begin{array}{c}\text { Mean } \\
\text { Square }\end{array}$ & F & p-value \\
\hline$N G$ & 74855.88 & 2 & 37427.9 & 536.29 & 0.00 \\
\hline$N P$ & 5555.54 & 2 & 2777.77 & 39.80 & 0.00 \\
\hline$P_{g}$ & 18183.81 & 2 & 9091.90 & 130.27 & 0.00 \\
\hline$N G^{*} N P$ & 3402.98 & 4 & 850.74 & 12.19 & 0.00 \\
\hline$N F^{*} P_{g}$ & 20513.94 & 4 & 5128.48 & 73.49 & 0.00 \\
\hline$N P^{*} P_{g}$ & 1244.71 & 4 & 311.18 & 4.46 & 0.001 \\
\hline$N P^{*} N{ }^{*} P_{g}$ & 1101.17 & 8 & 137.65 & 1.97 & 0.048 \\
\hline Error & 35801.90 & 513 & 69.79 & & \\
\hline Corrected Total & 749116 & 540 & & & \\
\hline
\end{tabular}



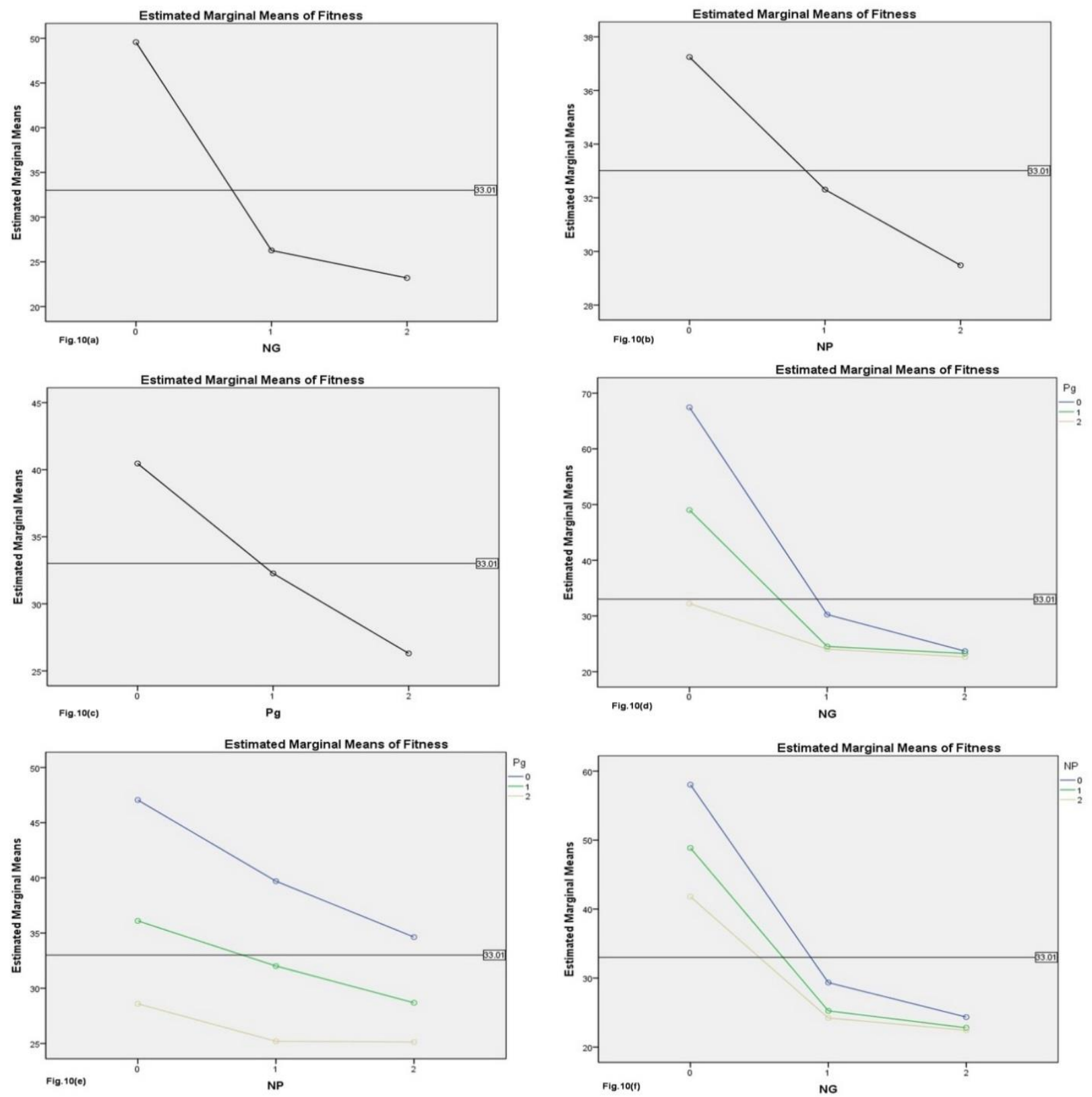

Figure 10. Main effects and Interactions of parameters

\subsubsection{Parameter tuning for TS, MCRW, GGA, SPSO}

Similarly, we also conducted parameter tuning for all benchmarking methods, namely, TS, MCRW, GGA, and SPSO. Three levels of each parameter for TS, MCRW, GGA, and SPSO are set (Table 5). For each method, the best-tuned parameters are highlighted. $T L$ means length of tabu list in TS. The random walk probability ranges from 0.05 to 0.15 because high probability may lead to higher frequency of walking into the infeasible solution space. The crossover rate is set to 0.7 to 0.9 whereas mutation rate is set to 0.02 to 0.1 . The parameters $w, c 1, c 2$ characterize the behavior of particles, $c 1+c 2<=4$, in this section, we set them ranging from 0.5 to 2 . Given that space limit, details can be disclosed upon request. 
Table 5. Full factorial design for all benchmarking algorithms

\begin{tabular}{|c|c|c|c|c|}
\hline \multicolumn{2}{|c|}{ level } & Low & Medium & High \\
\hline \multirow{2}{*}{ TS } & NG & 20 & 40 & 80 \\
\hline & TL & 8 & 12 & 16 \\
\hline \multirow{2}{*}{ MCRW } & NG & 100 & 400 & 800 \\
\hline & $\mathbf{P v}$ & 0.05 & 0.1 & 0.15 \\
\hline \multirow{5}{*}{ GGA } & NG & 40 & 80 & 160 \\
\hline & NP & 20 & 30 & 40 \\
\hline & Pc & 0.7 & 0.8 & 0.9 \\
\hline & $\mathbf{P v}$ & 0.02 & 0.05 & 0.1 \\
\hline & Pg & 0.3 & 0.5 & 0.8 \\
\hline \multirow{5}{*}{ SPSO } & NG & 40 & 80 & 160 \\
\hline & NP & 20 & 30 & 40 \\
\hline & C1 & 0.5 & 1 & 2 \\
\hline & $\mathrm{C2}$ & 0.5 & 1 & 2 \\
\hline & $\mathbf{W}$ & 0.3 & 0.6 & 0.9 \\
\hline
\end{tabular}

\subsubsection{Second Experiment}

Our GDPSO (with novel genetic operators) and DPSOs 1 to 3 (with common genetic operators) are compared in the second experiment. The three parameters are $N P=30, N G=40$, and $P_{g}=0.8$ as determined in the first experiment. The experiments of comparison among DPSOs are run 20 times to obtain the stability of algorithms indicating by the average value "Avg" and the minimal value "Min", as shown in table 6. All the above algorithms took nearly 2 min to run over 40 generations. The results indicate that our GDPSO can converge faster and obtain better solutions as compared with DPSO 1 to 3. Table 6 reports the number of constraint violations by different methods whereas Table 7 shows that GDPSO can significantly outperform other DPSO-based methods $(p<0.05)$. Therefore, our GDPSO with novel genetic operators is deemed to be more effective and efficient than DPSO with common genetic operators in minimizing the constraint violation of the EDCC timetabling problem. We also examine the convergence of each algorithm, which is indicated by the minimal cost of constraint violation in each generation of the algorithms. Figure 11 shows the convergence results of all algorithms solving the Monday case (the other five cases have similar results). 
Table 6. Number of constraint violation of different DPSO-based methods

\begin{tabular}{|c|c|c|c|c|c|c|c|c|}
\hline \multirow{2}{*}{ Alg } & \multicolumn{2}{|c|}{ GDPSO } & \multicolumn{2}{c|}{ DPSO1 } & \multicolumn{2}{c|}{ DPSO2 } & \multicolumn{2}{c|}{ DPSO3 } \\
\cline { 2 - 10 } Cases & Avg. & Min. & Avg. & Min. & Avg. & Min. & Avg. & Min. \\
\hline Mon & $\mathbf{2 3 . 5}$ & $\mathbf{1 8}$ & 120.2 & 105 & 122.4 & 113 & 107.6 & 98 \\
\hline Tue & $\mathbf{2 0 . 6}$ & $\mathbf{1 7}$ & 111.5 & 101 & 112.7 & 103 & 101 & 92 \\
\hline Wed & $\mathbf{2 5 . 8}$ & $\mathbf{2 3}$ & 125.7 & 116 & 128.9 & 119 & 112.8 & 104 \\
\hline Thu & $\mathbf{1 9 . 5}$ & $\mathbf{1 6}$ & 106.1 & 93 & 106.3 & 97 & 92.8 & 83 \\
\hline Fri & $\mathbf{2 5 . 4}$ & $\mathbf{2 2}$ & 129.5 & 124 & 130.4 & 115 & 117.4 & 107 \\
\hline Sat & $\mathbf{1 5 . 9}$ & $\mathbf{1 2}$ & 97 & 87 & 96.9 & 86 & 84.9 & 77 \\
\hline
\end{tabular}

Table 7. Two-sample t-test results among different DPSO-based methods

\begin{tabular}{|c|c|c|c|c|}
\hline P-value for t-test & GDPSO & DPSO1 & DPSO2 & DPSO3 \\
\hline GDPSO & - & $\mathbf{3 . 8 E}-117$ & $\mathbf{1 . 1 0 E - 1 1 3}$ & $\mathbf{1 . 2 9 E - 1 1 0}$ \\
\hline DPSO1 & & - & 0.44 & $1.45 \mathrm{E}-12$ \\
\hline DPSO2 & & & - & $4.36 \mathrm{E}-14$ \\
\hline
\end{tabular}

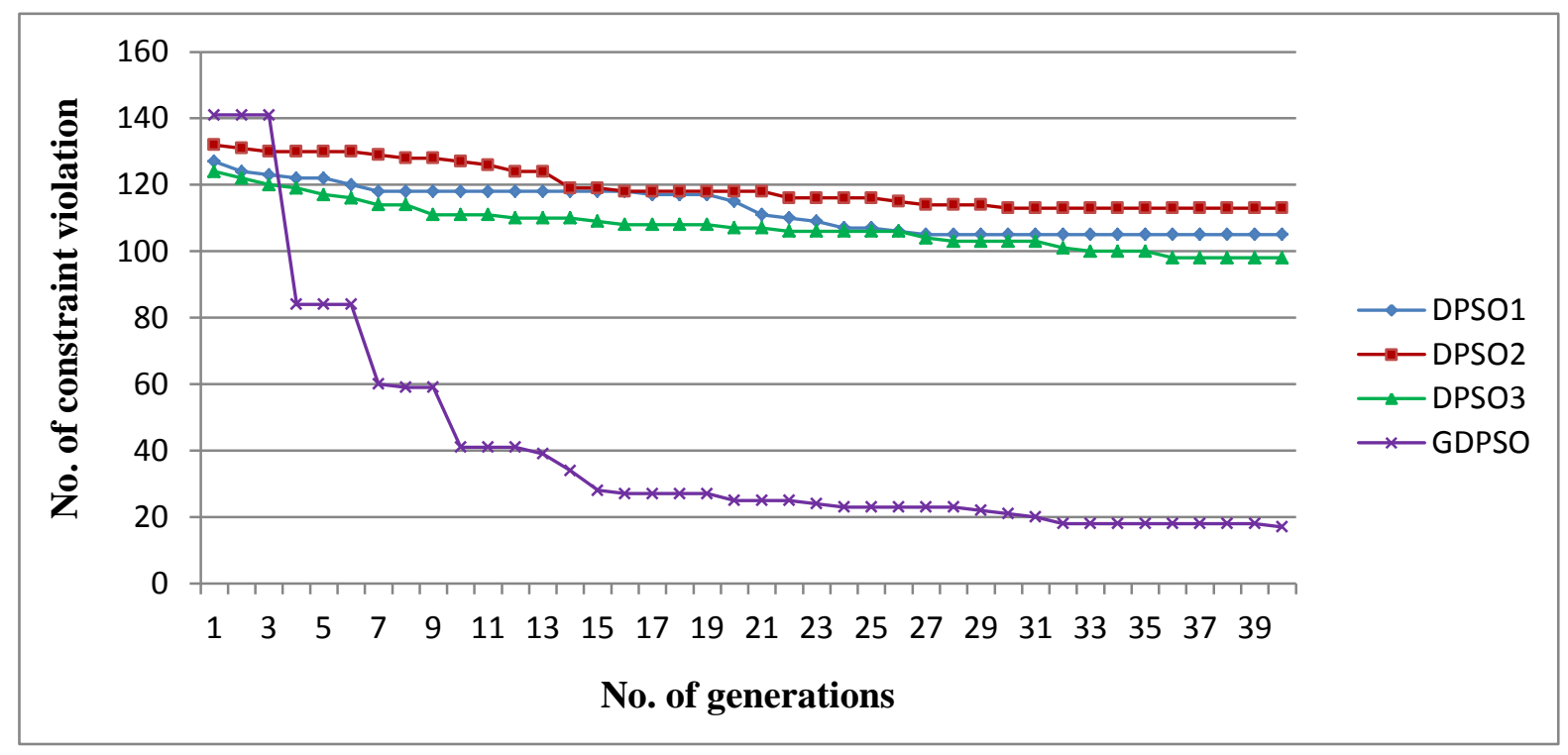

Figure 11. Convergence of DPSO with different genetic operators

\subsubsection{Third Experiment}

In this experiment, other fine-tuned benchmarking methods are compared with our fine-tuned GDPSO. All algorithms are assessed by the quality of performance, namely, the cost of constraint violations with the maximum computational time (e.g. $120 \mathrm{~s}$ ) permitted to solve the problems. Considering its randomness, the convergence of MCRW varies significantly for Monday case (Figure 12). Although the minimal violation of constraints could be achieved by MCRW, its searching process highly fluctuated. The convergence of all other algorithms is also shown in Figure 13. The figure shows the minimal cost of constraint violation in each generation of other 
algorithms when the Monday case is tested. The comparison results indicate that our GDPSO can converge faster and a better solution can be obtained within similar computational effort (i.e. 120 s). In fact, we have also done the experiments with 360s and find that the proposed GDPSO just need less than 120s to converge to a certain better solution (with 20 soft constraint violations) that can provide important information about constraints violations for HHC structure. Hence, 120s is considered as an appropriate computing time.

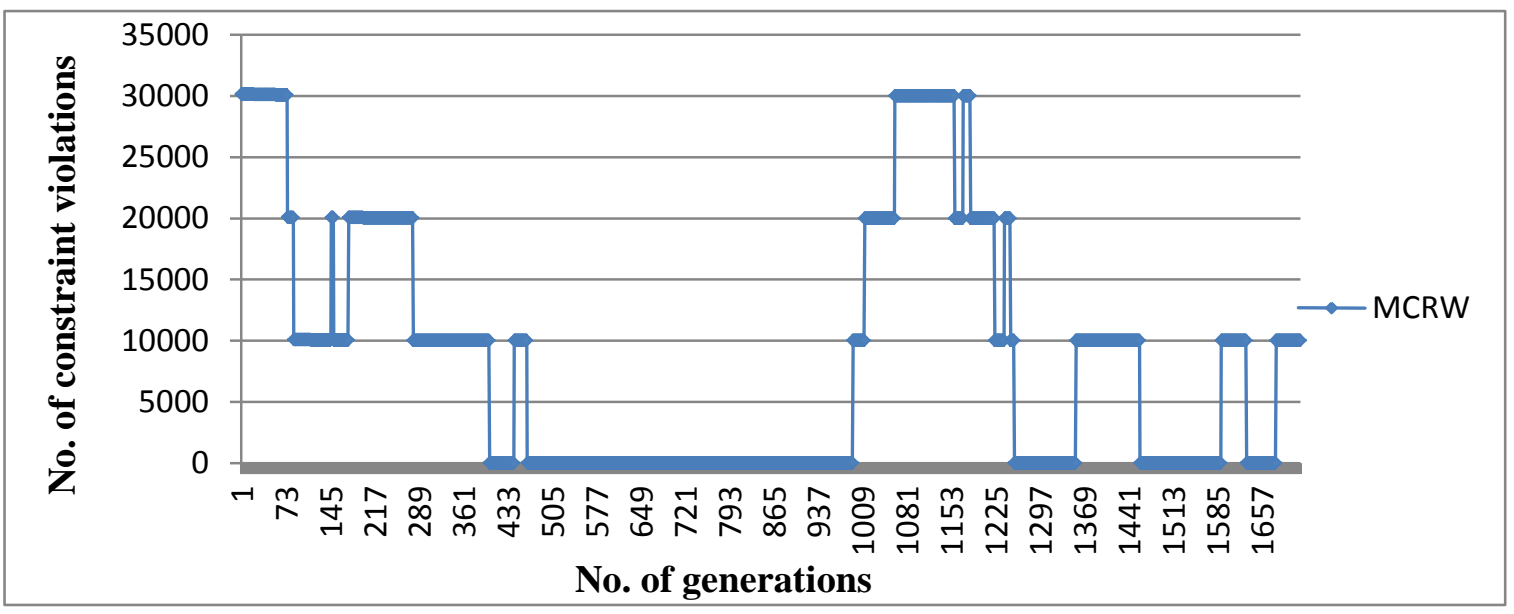

Figure 12. Convergence of MCRW

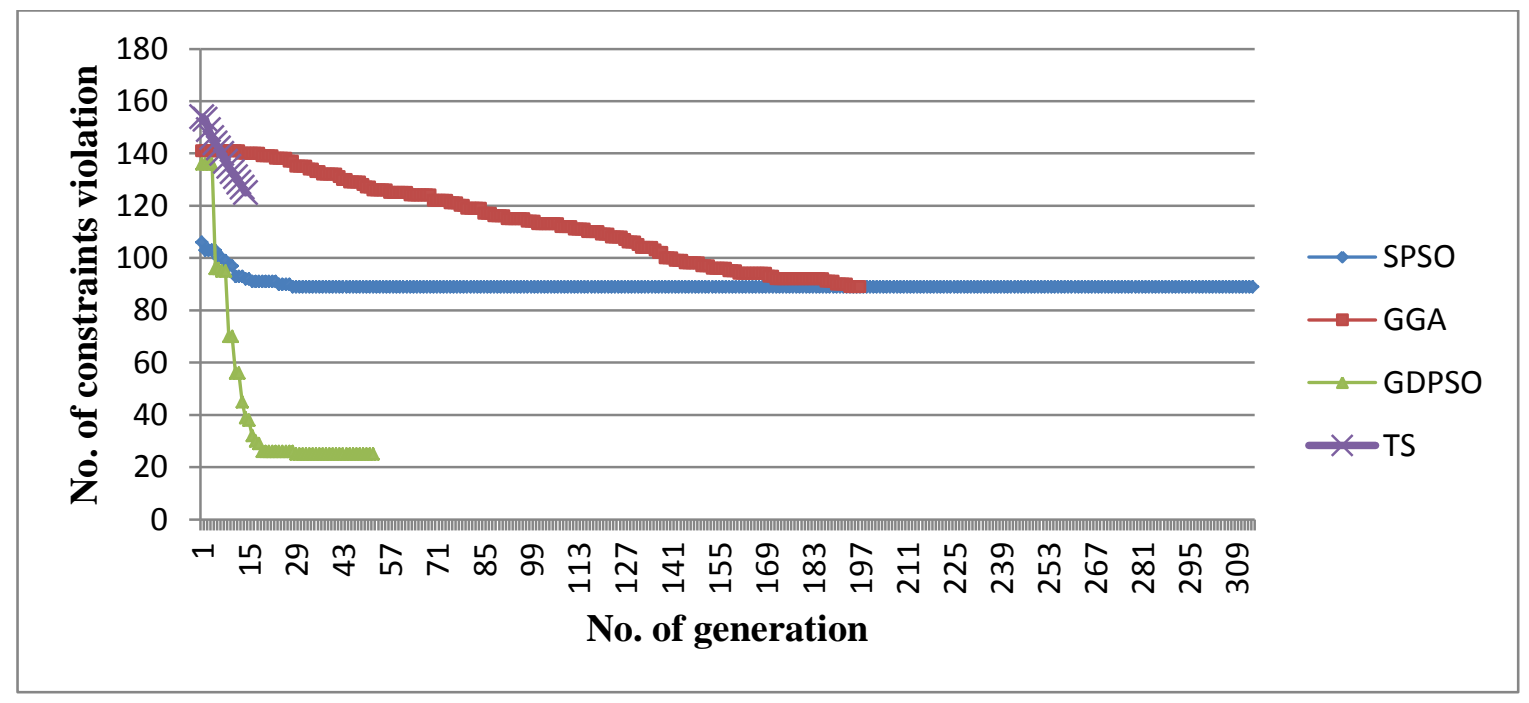

Figure 13. Convergence of algorithms

Tables 8 reports the comparison among these algorithms with fine-tuned parameters indicated in table 5. Column "Avg" indicates the average number of constraint violations across 20 runs obtained by each algorithm. Column "Min" indicates the minimum constraint violation obtained by each algorithm. Table 8 also illustrates that the GDPSO can significantly outperform the other four algorithms in terms of the average number of constraint violations. The average number of 
constraint violations of MCRW is the largest, because of its random walk scheme which may frequently violate hard constraints. The minimum constraint violation is obtained by MCRW rather than GDPSO, which indicates that MCRW sometimes gives a better result than GDPSO. However, MCRW is unstable because this method cannot always find feasible solutions in a few trials. Hence, we can conclude that GDPSO can perform much better than other algorithms in terms of stability and efficiency. Table 9 shows the average infeasibility value of 20 best solutions obtained by each algorithm subject to maximum computational time over 20 runs. Thus, only MCRW violates hard constraints more frequently than other algorithms.

Table 8. Performance of algorithms

\begin{tabular}{|c|c|c|c|c|c|c|c|c|c|c|}
\hline \multirow{2}{*}{ Alg } & \multicolumn{2}{|c|}{ SPSO } & \multicolumn{2}{c|}{ GGA } & \multicolumn{2}{c|}{ GDPSO } & \multicolumn{2}{c|}{ MCRW } & \multicolumn{2}{c|}{ TS } \\
\cline { 2 - 11 } & Avg & Min & Avg & Min & Avg & Min & Avg & Min & Avg & Min \\
\hline Mon & 97.4 & 89 & 81 & 70 & $\mathbf{2 3 . 5}$ & 20 & 8515 & 12 & 126.6 & 110 \\
\hline Tue & 90.1 & 74 & 71.2 & 60 & $\mathbf{1 9 . 3}$ & 17 & 9013.9 & 9 & 113.1 & 104 \\
\hline Wed & 102.8 & 93 & 87.3 & 79 & $\mathbf{2 6 . 5}$ & 22 & 7016.6 & 13 & 133.8 & 119 \\
\hline Thu & 86.1 & 77 & 64.4 & 58 & $\mathbf{1 8 . 6}$ & 16 & 7513.8 & 11 & 105.2 & 90 \\
\hline Fri & 101.5 & 93 & 93 & 85 & $\mathbf{2 6 . 1}$ & 22 & 9017.4 & 12 & 137.5 & 111 \\
\hline Sat & 80.1 & 67 & 55.7 & 45 & $\mathbf{1 5 . 7}$ & 13 & 9511.8 & 6 & 97.1 & 81 \\
\hline
\end{tabular}

Table 9. Average infeasibility value of solutions

\begin{tabular}{|c|c|c|c|c|c|c|c|}
\hline Hard Constraints & Cases & Mon & Tue & Wed & Thu & Fri & Sat \\
\hline \multirow{4}{*}{$\mathbf{C}_{1}$} & SPSO & 0 & 0 & 0 & 0 & 0 & 0 \\
\cline { 2 - 8 } & GGA & 0 & 0 & 0 & 0 & 0 & 0 \\
\cline { 2 - 8 } & GDPSO & 0 & 0 & 0 & 0 & 0 & 0 \\
\cline { 2 - 8 } & MCRW & 0.85 & 0.9 & 0.65 & 0.75 & 0.95 & 0.8 \\
\cline { 2 - 8 } & TS & 0 & 0 & 0 & 0 & 0 & 0 \\
\hline \multirow{4}{*}{$\mathbf{C}_{3}$} & SPSO & 0 & 0 & 0 & 0 & 0 & 0 \\
\cline { 2 - 8 } & GGA & 0 & 0 & 0 & 0 & 0 & 0 \\
\cline { 2 - 8 } & GDPSO & 0 & 0 & 0 & 0 & 0 & 0 \\
\cline { 2 - 8 } & MCRW & 0 & 0 & 0.05 & 0 & 0 & 0.1 \\
\cline { 2 - 8 } & TS & 0 & 0 & 0 & 0 & 0 & 0 \\
\hline
\end{tabular}

Table 10 shows the average objective value of solutions on each day and over a week. $C_{4}$ (time window constraints), $C_{6}$ (equipment capacity), $C_{7}$ (assistants capacity), and $C_{8}$ (room availability) are frequently violated. This result implies that the center can make a few improvements by considering the extension of room operating hours or enhancement of equipment capacity. In this connection, the center will be able to accommodate more users (quantity) and better meet their needs (quality). 
Table 10. Average number of violation of soft constraints

\begin{tabular}{|c|c|c|c|c|c|c|c|c|}
\hline $\begin{array}{c}\text { Soft } \\
\text { Constraints }\end{array}$ & Alg Cases & Mon & Tue & Wed & Thu & Fri & Sat & Total \\
\hline \multirow{5}{*}{$\mathbf{C}_{4}$} & SPSO & 8.7 & 10.15 & 10 & 6.55 & 9.4 & 8.7 & 53.5 \\
\hline & GGA & 14.5 & 14.3 & 17.45 & 13.15 & 11.55 & 20 & 90.95 \\
\hline & GDPSO & 1.05 & 0.6 & 1.6 & 0.5 & 0.45 & 1.35 & 5.55 \\
\hline & MCRW & 1.85 & 1.9 & 2.45 & 2.1 & 1.95 & 2.1 & 12.35 \\
\hline & TS & 25.7 & 21 & 24.95 & 19.65 & 19.25 & 29.3 & 139.85 \\
\hline \multirow{5}{*}{$\mathrm{C}_{5}$} & SPSO & 0 & 0 & 0 & 0 & 0 & 0 & 0 \\
\hline & GGA & 0 & 0 & 0 & 0 & 0 & 0 & 0 \\
\hline & GDPSO & 1.4 & 0.5 & 1.4 & 0.55 & 0.2 & 1.4 & 5.45 \\
\hline & MCRW & 0.3 & 0 & 0.35 & 0.15 & 0 & 0.15 & 0.95 \\
\hline & TS & 0 & 0.05 & 0 & 0 & 0 & 0.05 & 0.1 \\
\hline \multirow{5}{*}{$\mathrm{C}_{6}$} & SPSO & 0 & 0 & 0 & 0 & 0 & 0 & 0 \\
\hline & GGA & 0.99 & 0.66 & 1.14 & 0.714 & 0.48 & 1.08 & 5.05 \\
\hline & GDPSO & 3.33 & 2.95 & 3.49 & 2.73 & 2.29 & 3.78 & 18.55 \\
\hline & MCRW & 1.25 & 1.19 & 1.5 & 1.1 & 0.99 & 1.44 & 7.46 \\
\hline & TS & 1.35 & 1.21 & 1.28 & 0.9 & 0.63 & 1.63 & 6.99 \\
\hline \multirow{5}{*}{$\mathbf{C}_{7}$} & SPSO & 0 & 0 & 0 & 0 & 0 & 0 & 0 \\
\hline & GGA & 2.45 & 1.85 & 2.4 & 1.9 & 0.95 & 2.75 & 12.3 \\
\hline & GDPSO & 4.2 & 4.2 & 4 & 3.9 & 2.75 & 4.4 & 23.45 \\
\hline & MCRW & 2.35 & 2.65 & 2.85 & 2.9 & 1.85 & 2.65 & 15.25 \\
\hline & TS & 2.25 & 2.25 & 2.35 & 1.85 & 1.3 & 2.75 & 12.75 \\
\hline \multirow{5}{*}{$\mathrm{C}_{8}$} & SPSO & 43 & 38.7 & 45.23 & 38.55 & 34.35 & 45.15 & 244.98 \\
\hline & GGA & 28.48 & 24.63 & 29.65 & 21.63 & 19.05 & 31.05 & 154.48 \\
\hline & GDPSO & 1.75 & 1.075 & 2.7 & 1.375 & 1.55 & 1.85 & 10.3 \\
\hline & MCRW & 2.53 & 1.98 & 2.2 & 1.93 & 1.85 & 3.2 & 13.68 \\
\hline & TS & 44.78 & 40.45 & 48.7 & 38.1 & 35.25 & 47 & 254.28 \\
\hline \multirow{5}{*}{$\mathrm{C}_{9}$} & SPSO & 2.7 & 2.55 & 2.3 & 2.45 & 2 & 2.5 & 14.5 \\
\hline & GGA & 3.15 & 3.1 & 3.55 & 3.25 & 3.15 & 3.85 & 20.05 \\
\hline & GDPSO & 0 & 0.05 & 0.1 & 0 & 0.05 & 0.1 & 0.3 \\
\hline & MCRW & 0.45 & 0.6 & 0.55 & 0.35 & 0.35 & 0.3 & 2.6 \\
\hline & TS & 3.7 & 4 & 3.95 & 3.9 & 3.55 & 4.85 & 23.95 \\
\hline
\end{tabular}

\section{Conclusion and future research}

In this paper, Genetic-based Discrete Particle Swarm Optimization (GDPSO) is proposed to solve the EDCC timetabling problem, which is formulated as a weighted Max-CSP. The sensitivity analysis shows that the $N G, N P$, and $P_{g}$ do significantly affect the performance of GDPSO. Other benchmarking algorithms are fine-tuned and compared with GDPSO using our data sets. The comparison results suggest that GDPSO can outperform all the benchmarking algorithms in terms of stability and efficiency. In addition, GDPSO has a faster yet consistent rate of convergence and can obtain better results with shorter computing time. Based on the analysis of soft constraint violations (Table 10), the results suggest that the center can be improved by extending open hours of rooms and purchasing more equipment for activities. To the best of our knowledge, this study 
is one of the few attempts to explore the potential of evolutionary algorithms in solving Max-CSP. However, a few limitation of this study include that our problem is mainly defined with respect to the actual setting of the local day care center and several operating features are fixed. Thus, a more generalized EDCC problem will be studied to obtain a more generalized solution using our proposed GDPSO.

Our future work may focus more on the testing of GDPSO on larger Max-CSP, compared with the one reported in this paper. Moreover, the GDPSO search mechanism will be further investigated. In this study, the computational time for each example was nearly 2 min, which could be reduced to enhance the usefulness of our GDPSO. Finally, GDPSO can be used to solve other real-world problems, such as vehicle routing problems for home care visits and nurse scheduling problems in hospitals.

\section{Appendices}

The timetable for Monday obtained by GDPSO is presented to illustrate how users can be allocated to different timeslots of the same working day according to their needs. A total of 67 users have to complete several tasks on Monday, and the room capacity is 15 . Figure A1 shows the timetable for Room 1. Figures A2 shows the timetable for activities in Room 2 and activities $s_{34}$ to $s_{36}$ (group activity, walks, and baths).

The constraint violations are highlighted in different colors. The yellow color highlights the violation of equipment capacity and assistant capacity constraints, the red color highlights the violation of timeslot exclusion of room availability constraints, and the green color indicates the violation of the time window constraints of users. $P_{i}$ and $S_{j}$ denote the user id and activity id, respectively. The number of equipment of activity $s_{12}$ is 1 , such that the constraint of equipment capacity is violated in Room 1, as highlighted in yellow (Figure A1). Most violations can be observed in Room 2 including the activities $s_{34}$ to $s_{36}$. For example, $s_{27}$ to $s_{28}$ are activities that require an assistant. Considering that only one assistant is assigned to Room 2, several violations are highlighted in yellow (Figure A2). Room 2 is closed from 12:10 to 16:00; thus, this timeslot is unavailable for users, as indicated by red blocks in Figure A2. User id 65 (P65) cannot be assigned to timeslot 10:10 to 10:30 because this person reaches the center after 10:30. This 
violation is indicated by a green block in Figure A2. Regarding activities $s_{34}$ to $s_{36}$, several violations are also highlighted in yellow because similar equipment (only one unit is available) is required by more than one user at the same timeslot.

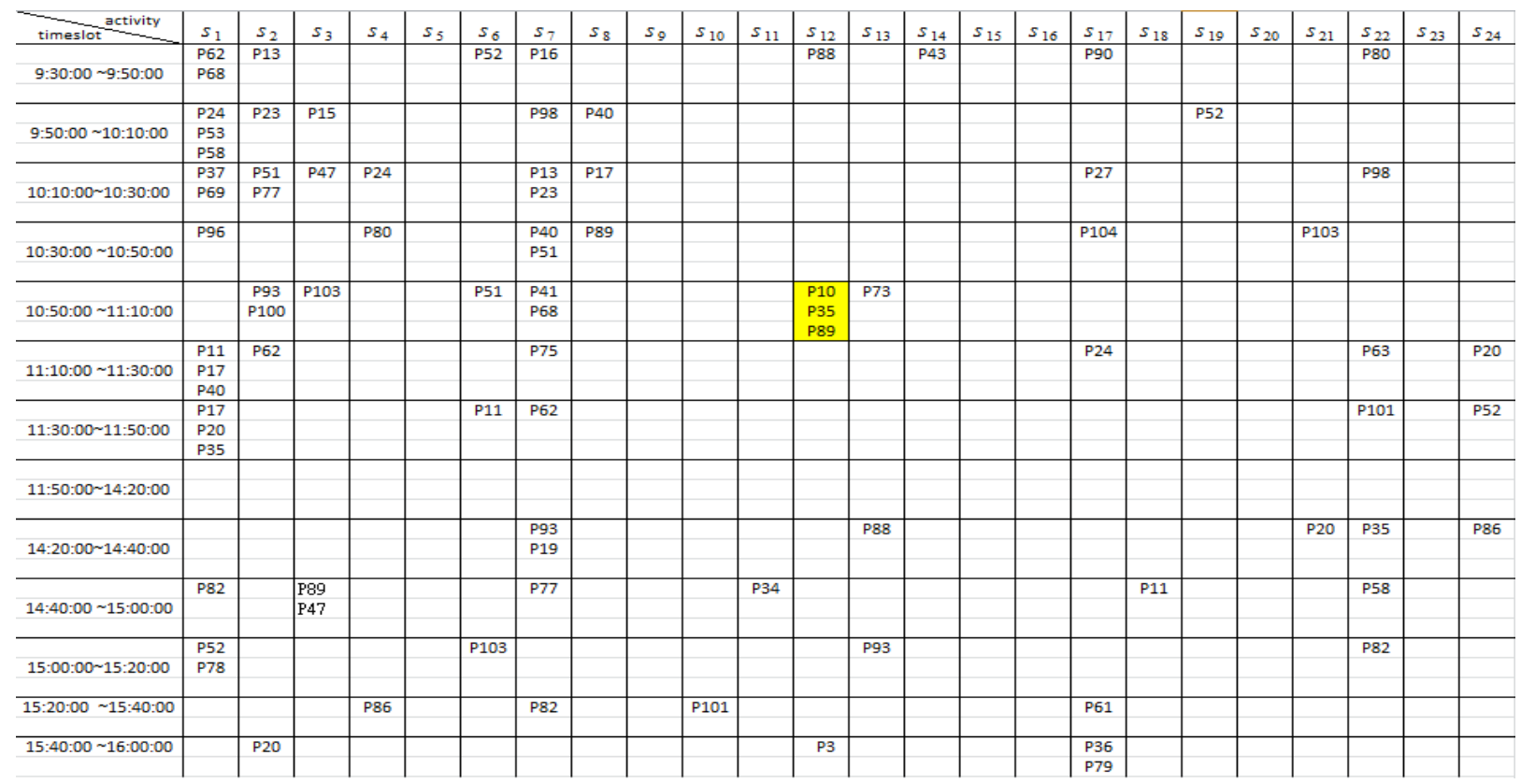

Figure A1. Timetable in Room 1

\begin{tabular}{|c|c|c|c|c|c|c|c|c|c|c|c|c|c|c|}
\hline timeslot activity & $s_{25}$ & & $s_{26}$ & & $s_{27}$ & $s_{28}$ & $s_{29}$ & $s_{30}$ & $s_{31}$ & $s_{32}$ & $s_{33}$ & $s_{34}$ & $s_{35}$ & $s_{36}$ \\
\hline & P29 & P33 & P6 & P18 & P24 & P68 & & & & & & & & \\
\hline \multirow{2}{*}{$9: 30: 00 \sim 9: 50: 00$} & $\mathrm{P} 40$ & P77 & P23 & P47 & & & & & & & & & & \\
\hline & P103 & & P53 & P69 & & & & & & & & & & \\
\hline \multirow[t]{3}{*}{ 9:50:00 10:10:00 } & P6 & P13 & P29 & P33 & & P80 & & & P48 & & & & & \\
\hline & P43 & P88 & P68 & P76 & & & & & P104 & & & & & \\
\hline & P90 & & & & & & & & & & & & & \\
\hline \multirow[t]{3}{*}{$10: 10: 00 \sim 10: 30: 00$} & P52 & P57 & P3 & P8 & & P6 & & $\mathrm{P} 65$ & P41 & P62 & & & P39 & \\
\hline & P60 & & P38 & P40 & & & & & & & & & & \\
\hline & & & P59 & P103 & & & & & & & & & & \\
\hline \multirow[t]{4}{*}{$10: 30: 00 \sim 10: 50: 00$} & $\mathrm{P} 23$ & P24 & & & P61 & P41 & P14 & & P27 & P47 & P57 & P62 & P59 & \\
\hline & P39 & P53 & & & & & & & & & & & & \\
\hline & P69 & P73 & & & & & & & & & & & & \\
\hline & P98 & & & & & & & & & & & & & \\
\hline \multirow[t]{4}{*}{$10: 50: 00 \sim 11: 10: 00$} & P18 & P19 & P20 & P34 & P17 & & & & & P65 & & P58 & P11 & P12 \\
\hline & P23 & P76 & P37 & P60 & P40 & & & & & & & P27 & P63 & P45 \\
\hline & & & & & P69,P90 & & & & & & & & P89 & \\
\hline & & & & & P79,P81 & & & & & & & & & \\
\hline \multirow[t]{3}{*}{$11: 10: 00 \sim 11: 30: 00$} & P3 & P8 & P19 & P41 & P77 & & P100 & & & & & & & P27 \\
\hline & P12 & P35 & P44 & P73 & & & & & & & & & & \\
\hline & P45 & P68 & P92 & & & & & & & & & & & \\
\hline \multirow[t]{3}{*}{ 11:30:00 11:50:00 } & P15 & P63,P93 & P10 & P88 & P82 & P12 & & & & & & FS2 F95 & & \\
\hline & P78 & P79 & P96 & & & P27 & & & & & & & & \\
\hline & P83 & P92 & & & & & & & & & & & & \\
\hline \multirow[t]{3}{*}{ 11:50:00 12:10:00 } & P61 & P82 & P11 & P35 & P57 & & P45 & P17 & P80 & P10 & P81 & & & P73 \\
\hline & P96 & P100 & P63 & P78 & & & & & & & & & & \\
\hline & & & P83 & & & & & & & & & & & \\
\hline \multirow[t]{2}{*}{$12: 10: 00 \sim 12: 30: 00$} & & & & & & & & & & & & & & P97 \\
\hline & & & & & & & & & & & & & & \\
\hline \multirow[t]{3}{*}{$12: 40: 00 \sim 13: 00: 00$} & & & & & & & & & & & & & & P10,P104 \\
\hline & & & & & & & & & & & & & & P34 \\
\hline & & & & & & & & & & & & & & P82 \\
\hline $13: 00: 00 \sim 13: 20: 00$ & & & & & P62 & & & & & & & \begin{tabular}{|l|} 
F44 P104 \\
\end{tabular} & & \\
\hline \multirow{2}{*}{\multicolumn{9}{|c|}{$\begin{array}{l}13: 20: 00 \sim 13: 40: 00 \\
13: 40: 00 ~ 14: 00: 00\end{array}$}} & & P101 & & & & \\
\hline & & & & & & & & & P81 & & & & & \\
\hline \multicolumn{15}{|l|}{$14: 00: 00 \sim 14: 40: 00$} \\
\hline \multicolumn{15}{|l|}{$14: 40: 00 \sim 15: 00: 00$} \\
\hline $15: 00: 00 \sim 15: 20: 00$ & & & & & & & & & & & & & P51 & \\
\hline \multicolumn{15}{|l|}{$15: 20: 00 \sim 15: 40: 00$} \\
\hline $15: 40: 00 \sim 16: 00: 00$ & & & & & & & & & & P89 & & & & \\
\hline
\end{tabular}

Figure A2. Timetable in Room 2 


\section{Reference}

[1] Christensen K, Doblhammer G, Rau R, Vaupel JW. Ageing populations: The challenges ahead. The Lancet. 2009;374(9696):1196-208

[2] Reinhardt UE. Does the aging of the population really drive the demand for health care? Health Affairs. 2003;22(6):27-39

[3] Solos IP, Tassopoulos IX, Beligiannis GN. A generic two-phase stochastic variable neighborhood approach for effectively solving the nurse rostering problem. Algorithms. 2013;6(2):278-308.

[4] Della Croce F, Salassa F. A variable neighborhood search based matheuristic for nurse rostering problems. Ann Oper Res. 2014;218(1):185-99.

[5] Santos HG, Toffolo TAM, Gomes RAM, Ribas S. Integer programming techniques for the nurse rostering problem. Ann Oper Res. 2014:1-27.

[6] Burke EK, Curtois T. New approaches to nurse rostering benchmark instances. European Journal of Operational Research. 2014;237(1):71-81.

[7] Tassopoulos IX, Solos IP, Beligiannis GN. A two-phase adaptive variable neighborhood approach for nurse rostering. Computers \& Operations Research. 2015;60:150-69.

[8] Cacchiani V, Toth P. Nominal and robust train timetabling problems. European Journal of Operational Research. 2012;219(3):727-37

[9] Jat SN, Yang SX. A hybrid genetic algorithm and tabu search approach for post enrolment course timetabling. Journal of Scheduling. 2011;14(6):617-37.

[10] Shiau DF. A hybrid particle swarm optimization for a university course scheduling problem with flexible preferences. Expert Systems with Applications. 2011;38(1):235-48.

[11] Yang Sx, Jat SN. Genetic algorithms with guided and local search strategies for university course timetabling. IEEE Transactions on Systems, Man, and Cybernetics, Part C: Applications and Reviews. 2011;41(1):93-106

[12] Ceschia S, Di Gaspero L, Schaerf A. Design, engineering, and experimental analysis of a simulated annealing approach to the post-enrolment course timetabling problem. Computers \& Operations Research. 2012;39(7):1615-24.

[13] Tassopoulos IX, Beligiannis GN. A hybrid particle swarm optimization based algorithm for high school timetabling problems. Applied Soft Computing. 2012;12(11):3472-89.

[14] Chen RM, Shih HF. Solving university course timetabling problems using constriction particle swarm optimization with local search. Algorithms. 2013;6(2):227-44.

[15] Alzaqebah M, Abdullah S. Hybrid bee colony optimization for examination timetabling problems. Computers \& Operations Research. 2015;54(0):142-54.

[16] Freuder EC, Wallace RJ. Partial constraint satisfaction. Artificial Intelligence. 1992;58(1):21-70

[17] Normand JM, Goldsztejn A, Christie M, Benhamou F. A branch and bound algorithm for numerical Max-CSP. Constraints. 2010;15(2):213-37.

[18] Minton S, Johnston MD, Philips AB, Laird P. Minimizing conflicts: A heuristic repair method for constraint satisfaction and scheduling problems. Artificial Intelligence. 1992;58(1):161-205

[19] Galinier P, Hao JK. Tabu search for maximal constraint satisfaction problems. In: Smolka G, (Ed.). Principles and Practice of Constraint Programming-CP97: Springer; 1997. p. 196-208 
[20] Bouamama S, Jlifi B, Ghédira K. D2G2A: A distributed double guided genetic algorithm for Max_CSPs. In: Palade V, Howlett RJ, Jain L, (Eds.) Knowledge-Based Intelligent Information and Engineering Systems: Springer; 2003. p. 422-9

[21] Khadhraoui A, Bouamama S. A new hybrid distributed double guided genetic swarm algorithm for optimization and constraint reasoning: Case of Max-CSPs. International Journal of Swarm Intelligence Research. 2012;3(2):63-74.

[22] Tseng CT, Liao CJ. A discrete particle swarm optimization for lot-streaming flowshop scheduling problem. European Journal of Operational Research. 2008;191(2):360-73

[23] Zhang CS, Sun JG, Zhu XJ, Yang QY. An improved particle swarm optimization algorithm for flowshop scheduling problem. Information Processing Letters. 2008;108(4):204-9.

[24] Ponnambalam SG, Jawahar N, Chandrasekaran S. Discrete particle swarm optimization algorithm for flowshop scheduling. In: Lazinica A, (Ed.). Particle Swarm Optimization: INTECH Open Access Publisher; 2009.

[25] Wang XP, Tang LX. A discrete particle swarm optimization algorithm with self-adaptive diversity control for the permutation flowshop problem with blocking. Applied Soft Computing. 2012;12(2):652-62

[26] Shao XY, Liu WQ, Liu Q, Zhang CY. Hybrid discrete particle swarm optimization for multi-objective flexible job-shop scheduling problem. The International Journal of Advanced Manufacturing Technology. 2013:1-17

[27] Goksal FP, Karaoglan I, Altiparmak F. A hybrid discrete particle swarm optimization for vehicle routing problem with simultaneous pickup and delivery. Computers \& Industrial Engineering 2012.

[28] Kechagiopoulos PN, Beligiannis GN. Solving the urban transit routing problem using a particle swarm optimization based algorithm. Applied Soft Computing. 2014;21:654-76.

[29] Ho TK, Tsang CW, Ip KH, Kwan KS. Train service timetabling in railway open markets by particle swarm optimisation. Expert Systems with Applications. 2012;39(1):861-8.

[30] Bratton D, Kennedy J. Defining a standard for particle swarm optimization. the 4th Swarm Intelligence Symposium (SIS'07). Honolulu, Hawaii, USA: IEEE; 2007. p. 120-7.

[31] Burke EK, Kingston J. Applications to timetabling. In: Gross JL, Yellen J, (Eds.) Handbook of graph theory: CRC Press; 2004. p. 445.

[32] Pillay N. A survey of school timetabling research. Ann Oper Res. 2014;218(1):261-93.

[33] MirHassani SA, Habibi F. Solution approaches to the course timetabling problem. Artificial Intelligence Review. 2013;39(2):133-49.

[34] Qu R, Burke EK, McCollum B, Merlot LTG, Lee SY. A survey of search methodologies and automated system development for examination timetabling. Journal of scheduling. 2009;12(1):55-89.

[35] Ribeiro CC. Sports scheduling: Problems and applications. International Transactions in Operational Research. 2012;19(1-2):201-26

[36] Cacchiani V, Caprara A, Toth P. Scheduling extra freight trains on railway networks. Transportation Research Part B: Methodological. 2010;44(2):215-31.

[37] Liebchen C, Möhring R. The modeling power of the periodic event scheduling problem: Railway timetables - and beyond. In: Geraets F, Kroon L, Schoebel A, Wagner D, Zaroliagis C, (Eds.) Algorithmic Methods for Railway Optimization: Springer Berlin Heidelberg; 2007. p. 3-40. 
[38] Phillips AE, Waterer H, Ehrgott M, Ryan DM. Integer programming methods for large-scale practical classroom assignment problems. Computers \& Operations Research. 2015;53:42-53.

[39] He F, Qu R. A constraint programming based column generation approach to nurse rostering problems. Computers \& Operations Research. 2012;39(12):3331-43.

[40] Eberhart R, Kennedy J. A new optimizer using particle swarm theory. the 6th International Symposium on Micro Machine and Human Science (MHS '95). Nagoya, Japan: IEEE; 1995. p. $39-43$

[41] Akjiratikarl C, Yenradee P, Drake PR. An improved particle swarm optimization algorithm for care worker scheduling. Industrial Engineeering \& Management Systems. 2008;7(2):171-81.

[42] Liu B, Wang L, Jin YH. An effective PSO-based memetic algorithm for flow shop scheduling. IEEE Transactions on Systems, Man, and Cybernetics, Part B: Cybernetics. 2007;37(1):18-27

[43] Zhang H, Li XD, Li H, Huang FL. Particle swarm optimization-based schemes for resource-constrained project scheduling. Automation in Construction. 2005;14(3):393-404.

[44] Jonsson P, Krokhin A, Kuivinen F. Ruling out polynomial-time approximation schemes for hard constraint satisfaction problems. In: Diekert V, Volkov M, Voronkov A, (Eds.) Computer Science - Theory and Applications: Springer Berlin Heidelberg; 2007. p. 182-93.

[45] Sharapov RR. Genetic algorithms: Basic ideas, variants and analysis. In: Obinata G, Dutta A, (Eds.) Vision Systems: Segmentation and Pattern Recognition: INTECH Open Access Publisher; 2007.

[46] Montgomery DC. Design and analysis of experiments: Wiley New York; 1984. 